\title{
Cantor Spectrum and Singular Continuity for a Hierarchical Hamiltonian *
}

\author{
Hervé Kunz ${ }^{1}$, Roberto Livi ${ }^{1, \star \star}$, and András Sütö ${ }^{2}$ \\ ${ }^{1}$ Institut de Physique Théorique, Ecole Polytechnique Fédérale de Lausanne, \\ CH-1015 Ecublens, Switzerland \\ ${ }^{2}$ Institut de Physique Théorique, Université de Lausanne, BSP, CH-1015 Lausanne, Switzerland
}

\begin{abstract}
We study the spectrum of the Hamiltonian $H$ on $l_{2}(\mathbb{Z})$ given by $(H \psi)(n)=\psi(n+1)+\psi(n-1)+V(n) \psi(n)$ with the hierarchical (ultrametric) potential $V\left(2^{m}(2 l+1)\right)=\lambda\left(1-R^{m}\right) /(1-R)$, corresponding to 1-, 2-, and 3-dimensional Coulomb potentials for $0<R<1, R=1$ and $R>1$, respectively, in a suitably chosen valuation metric. We prove that the spectrum is a Cantor set and gaps open at the eigenvalues $e_{n}(1)<e_{n}(2)<\ldots<e_{n}\left(2^{n}-1\right)$ of the Dirichlet problem $H \psi=E \psi, \psi(0)=\psi\left(2^{n}\right)=0, n \geqq 1$. In the gap opening at $e_{n}(k)$ the integrated density of states takes on the value $k / 2^{n}$. The spectrum is purely singular continuous for $R \geqq 1$ when the potential is unbounded, and the Lyapunov exponent $\gamma$ vanishes in the spectrum. The spectrum is purely continuous for $R<1$ in $\sigma(H) \cap[-2,2]$ and $\gamma=0$ here, but one cannot exclude the presence of eigenvalues near the border of the spectrum. We also propose an explicit formula for the Green's function.
\end{abstract}

\section{Table of Contents}

1. Introduction . . . . . . . . . . . . . . . . . . . . . . . . . . . 644

2. The Potential: Ultrametric Properties and Limit Periodicity . . . . . . . . . 646

3. Renormalization Group Transformation. The Trace Equation . . . . . . . . . 650

4. Asymptotic Behaviour of the Sequence of Traces . . . . . . . . . . . . . 654

5. Locating the Spectrum . . . . . . . . . . . . . . . . . . . . . . . 658

6. Cantor Spectrum, IDS and Gap Labelling . . . . . . . . . . . . . . . . 665

7. The Lyapunov Exponent . . . . . . . . . . . . . . . . . . . . . . . 672

8. Asymptotic Behaviour of the Gap-Edges States . . . . . . . . . . . . . . . 674

9. An Explicit Formula for the Green's Function . . . . . . . . . . . . . . . 675

* Work supported by the Fonds National Suisse de la Recherche Scientifique, Grant No. 2.042-0.86 (H.K. and R.L.) and 2.483-0.87 (A.S.)

$\star \star$ On leave from the Dipartimento di Fisica, Università degli Studi di Firenze, Largo E. Fermi 2, I-50125 Firenze, Italy 


\section{Introduction}

In the last years much attention has been devoted to the study of diffusion on random and hierarchical structures [1-6]. The peculiar behaviour for the diffusive problems has raised an interest in analyzing the spectral properties of Schrödinger operators with hierarchical, self-similar potentials. Various models have been proposed and studied both rigorously [6] and numerically [7]. General evidence of new interesting features for these spectra has been obtained, related to their Cantor-set like structure and scaling properties [6-11]. In particular, it seemed plausible to conjecture that a wide class of such hierarchical models could be characterized by a singular continuous spectrum.

Hierarchical potentials are a kind of almost periodic functions. Based on the considerable knowledge accumulated about almost periodic Schrödinger operators (see $[12,13]$ for two recent reviews), one indeed expects Cantor spectra for hierarchical Hamiltonians. Also, there is a general experience about singular continuous spectra $[14,15]$, and another self-similar Schrödinger operator, the Fibonacci Hamiltonian has, in fact, a singular continuous Cantor spectrum $[16,17]$.

In the present work our aim is to verify the expectations about Cantor spectrum and singular continuity for a hierarchical model. We discuss the spectral properties of the one-dimensional discrete Schrödinger operator

$$
(H \psi)(n)=\psi(n+1)+\psi(n-1)+V(n) \psi(n)
$$

with the hierarchical potential

$$
V(n)=\lambda f(\operatorname{ord}(n)) \text {. }
$$

Here $\lambda$ is a real number, $f$ is a real-valued function and ord $(n)$, the order of $n$, is the largest non-negative integer $m$ such that $2^{m}$ is a divisor of $n$. For a reason which becomes clear later on we choose a particular form for $f(\mathrm{cf} .[10,11])$,

$$
f(m)=\sum_{k=0}^{m-1} R^{k}
$$

where $R$ is a positive constant. We consider both $R<1$ and $R \geqq 1$ : in the latter case $V(n)$ is unbounded. The potential is even because ord $(-n)=\operatorname{ord}(n)$. For $R<1, H$ is defined on bi-infinite sequences $\{\psi(n)\}_{n=-\infty}^{\infty}$, but for $R \geqq 1$, it can be restricted to the half-line $n \geqq 1$ with boundary condition $\psi(0)=0$. Indeed, ord $(0)=\infty$, so that Eqs. (1.1) and (1.2) imply

$$
V(0)=\lambda \lim _{m \rightarrow \infty} f(m)= \begin{cases}\frac{\lambda}{1-R}, & R<1, \\ \infty, & R \geqq 1 .\end{cases}
$$

$V(0)=\infty$ means that the domain of $H$ is in the subspace $\psi(0)=0$. For $R \geqq 1$ we will understand, therefore, that $H$ is defined on the sequences $\{\psi(n)\}_{n=1}^{\infty}$ with boundary condition $\psi(0)=0$. Due to $V(-n)=V(n)$, the selection of the negative half-line would yield the same result. Throughout the whole paper we reserve the notation $H$ for the operator defined above. We will admit a rank one perturbation of $H$ yielding $H^{\prime}$ which differs from $H$ in the value of $V(0)$. Now $\sigma_{\text {ess }}\left(H^{\prime}\right)=\sigma(H)$, provided that $\sigma(H)$ contains no isolated points, which we eventually prove. 
We show that for all $R>0$ the spectrum $\sigma(H)$ is a Cantor set: a closed nowhere dense set without isolated points. According to any numerical evidence this Cantor set has a positive Lebesgue measure if $R<1$. Recently Bellissard [13] announced that the spectrum is purely singular continuous and of zero Lebesgue measure for $R>2$.

For $R \geqq 1$ we prove that $\sigma(H)$ and, in fact, $\sigma_{\text {ess }}\left(H^{\prime}\right)$ is purely continuous. Recently Simon and Spencer [26] proved that discrete Schrödinger operators (1.0) with unbounded potentials in both directions cannot have any absolutely continuous spectrum. Using this result we obtain that $\sigma(H)$ is purely singular continuous for $R \geqq 1$. The spectral measure of $H^{\prime}$ on $\sigma_{\text {ess }}\left(H^{\prime}\right)=\sigma(H)$ is also purely singular continuous, thus the singular continuity of $\sigma(H)$ has nothing to do with the decoupling at the site 0 . If $0<R<1$, then $V$ is limit periodic and one would expect a purely absolutely continuous spectrum. However, $\sigma(H)$ contains a special set $S$ with the following properties. $H \psi=E \psi$ with $E \in S$ may have a unique (up to factor) polynomially bounded solution, probably in $l_{2}(\mathbb{Z})$. $H^{\prime} \psi=E \psi$ with $E \in S$ has no polynomially bounded solution and $\sigma_{\text {ess }}\left(H^{\prime}\right)$ is purely continuous. The set $S$ is distant from a large part of $\sigma(H)$, e.g. $S \cap[-2,2]=\emptyset$, and $H$ cannot have eigenvalues outside $S$.

We obtain a partial (maybe complete) gap labelling. An extension from continuous almost periodic functions yields $\left\{k / 2^{n} \mid n \geqq 0, k=1, \ldots, 2^{n}\right\}$ as the frequency module of $V$. For continuous almost periodic potentials the integrated density of states (IDS) takes on values from the frequency module of the potential; however, this may be true if the potential is discontinuous [25]. Now our potential is singular in a certain sense (see Sect. 2), we nevertheless know: $H$ has a set of spectral gaps $\left\{I_{n k} \mid n \geqq 0,1 \leqq k \leqq 2^{n}\right\}$ with relative positions identical to those of the gaps of the classical triadic Cantor set. The IDS takes on the value $(2 k-1) / 2^{n+1}$ in $I_{n k}$, the same as the Cantor function in the corresponding gap of the classical Cantor set. $I_{n k}$ is bordered from below $(\lambda>0)$ or from above $(\lambda<0)$ by $E_{n}(k)$, the $k^{\text {th }}$ zero of $\tau_{n}(E)$, the trace of the transfer matrix over the interval $\left[1,2^{n}\right]$. For $R<1$, in the interval $[-2,2]$ all the gaps belong to the set $\left\{I_{n k}\right\}$.

We prove that the Lyapunov exponent exists and is zero in the spectrum for $R \geqq 1$, and the same holds true outside the set $S$ for $R<1$. A more detailed study is done for the states belonging to the gap edges $E_{n}(k)$. For these energies there is a unique (up to factor) periodic solution $\psi^{0}\left(\psi^{0}(0)=0\right)$. The other solutions increase linearly if $R<2$, as $x \log _{2} x$ if $R=2$ and as $x^{\log _{2} R}$ if $R>2$. In fact, we find this last behaviour for a periodic subset of sites $x$ but we believe this characterizes the envelope of the nonperiodic solutions.

All these results follow from a remarkable property of renormalization of the equation $H \psi=E \psi$, due to the form (1.1) of the potential. This, together with the choice (1.2) leads to an autonomous nonlinear recurrence equation for the traces $\tau_{n}$, similar to the one existing for the Fibonacci Hamiltonian [16-19] and exploited already in $[10,11]$. The spectral variable $E$ enters the recurrence via the initial condition and determines the asymptotic behaviour of $\tau_{n}$ as $n$ goes to infinity. We show that for any $E \in \mathbb{R}$ either $\left\{\tau_{n}\right\}$ is bounded or $\tau_{n} \rightarrow+\infty$ and $\tau_{n} / \tau_{n-1}^{2} \rightarrow R$ (if $R<1)$ or $\tau_{n} \rightarrow+\infty$ and $\tau_{n} / \tau_{n-1}^{2} \rightarrow \max \{1, R\}$. In the first two cases $E$ is in the spectrum, in the third case it is in the resolvent set. The second case specifies the set $S$. 
In order to motivate the reader to continue his lecture-or to spare him to do so - we sketch here the proof that for any $R>0$ the spectrum is a Cantor set:

(i) There are no isolated points in the spectrum. For $R<1$ this comes from the limit-periodicity of $V(n)$ while for $R \geqq 1$ the spectrum is purely continuous.

(ii) The eigenvalues of the Dirichlet problems $H \psi=E \psi, \psi(0)=\psi\left(2^{n}\right)=0$ for $n \geqq 1$ are dense in $\sigma(H)$ and they are at the border of spectral gaps of $H$. Therefore the gaps are dense everywhere.

Thus $\sigma(H)$ is a closed nowhere dense nonempty set without isolated points: it is a Cantor set. The absence of eigenvalues for $R \geqq 1$ and also for $R<1$ in a large part of the spectrum follows from a detailed analysis of the renormalization map.

The rest of the paper is organized as follows. In the next section we discuss the potential (1.1). We show that it is limit periodic for $R<1$. It can be written as a $d$-dimensional Coulomb potential in the valuation metric of 2 -adic numbers with $d$ varying with $R$. The elements of the hull of the potential are labelled by the 2 -adic integers. Section 3 presents the renormalized Schrödinger equation and the recurrence equation for the traces $\tau_{n}$. The asymptotic behaviour, as $n$ goes to infinity, of the sequence $\tau_{n}$ is analyzed in Sect. 4 . Sections 5 and 6 contain the main results of the paper: the determination of the spectrum via the asymptotics of $\left\{\tau_{n}\right\}$ and the results on the spectral measures in Sect. 5, the Cantor property, IDS and gap labelling in Sect. 6. Sections 7 and 8 deal with the asymptotic behaviour of the wave functions: they contain respectively the results on the Lyapunov exponent and on the gap-edge states. In Sect. 9 we give an explicit formula for the Green's function.

\section{The Potential: Ultrametric Properties and Limit Periodicity}

A bi-infinite sequence $V=\{V(n)\}_{-\infty}^{\infty}$ is called limit periodic if there exist periodic sequences $V_{N}=\left\{V_{N}(n)\right\}_{n=-\infty}^{\infty}$ tending uniformly to $V$ :

$$
\left\|V-V_{N}\right\|_{\infty}=\sup _{n}\left|V(n)-V_{N}(n)\right| \rightarrow 0 \quad \text { as } \quad N \rightarrow \infty \text {. }
$$

Consider now the potential (1.1). Since

$$
\operatorname{ord}\left(l \cdot 2^{n}+k\right)=\operatorname{ord}(k) \quad \text { if } \quad k=1,2, \ldots, 2^{n}-1,
$$

we have

$$
V\left(l \cdot 2^{n}+k\right)=V(k), \quad k=1,2, \ldots, 2^{n}-1
$$

for any $l \in \mathbb{Z}$ and $n \geqq 1$ integer. Choose $V_{N}$ to be periodic with period $2^{N}$ and

$$
V_{N}(n)=V(n), \quad n=1,2, \ldots, 2^{N} .
$$

Then $V_{N}$ differs from $V$ only at the sites $n=l \cdot 2^{N}, l \in \mathbb{Z}$. Therefore

$$
\begin{aligned}
\left\|V-V_{N}\right\|_{\infty} & =\sup _{l \in \mathbb{Z}}\left|V\left(l \cdot 2^{N}\right)-V\left(2^{N}\right)\right| \\
& =|\lambda| \sup _{l \in \mathbb{Z}}\left|f\left(\operatorname{ord}\left(l \cdot 2^{N}\right)\right)-f(N)\right| \\
& =|\lambda| \sup _{n \geqq N}|f(n)-f(N)|,
\end{aligned}
$$


where we used Eqs. (2.1) and (2.2), ord $(0)=\infty$ and $V(n)=V(-n)$. With the choice (1.2) for $f$ and for $R<1$,

$$
\left\|V-V_{N}\right\|_{\infty}=|\lambda| R^{N} /(1-R) \rightarrow 0 \quad \text { as } \quad N \rightarrow \infty
$$

so that $V$ is limit periodic.

However, $V$ is a rather peculiar sequence. It is singular in the sense that the values form a discrete set with a unique accumulation point: $\lambda /(1-R)$ if $R<1$ and $\infty$ if $R \geqq 1$. On the other hand, $V(n)$ can be viewed as a continuous function: To this end, the topology has to be changed. This will lead us to the hull of the potential. In what follows, we use definitions and basic results from the theory of $p$-adic numbers. For a general reference see [20].

Given some real numbers $s>1$, one can introduce a map

$$
1 \cdot l_{s}: \mathbb{Z} \rightarrow \mathbb{R}^{+}
$$

by the definition

$$
|n|_{s}=s^{-\operatorname{ord}(n)},
$$

where $2^{\operatorname{ord}(n)}$ is the highest power of 2 dividing $n .|\cdot|_{s}$ is called a valuation, $|\cdot|_{2}$ is the standard valuation. The valuation has properties similar to the absolute value:

1. $|n|_{s} \geqq 0$ with equality iff $n=0$,

2. $|-n|_{s}=|n|_{s}$,

3. $|n+m|_{s} \leqq \max \left\{|n|_{s},|m|_{s}\right\}$,

4. $|n m|_{s}=|n|_{s}|m|_{s}$.

Property 3 implies $|n+m|_{s} \leqq|n|_{s}+|m|_{s}$. Valuations with Property 3 are called nonarchimedian or ultrametric. $|\cdot|_{s}$ induces a metric by $d(n, m)=|n-m|_{s}$. The metric $\operatorname{space}\left(\mathbb{Z},|\cdot|_{s}\right)$ is precompact. By completing $\mathbb{Z}$ with respect to $|\cdot|_{s}$ with any $s>1$ one obtains the ring of 2-adic integers, $I_{2}$, satisfying Properties 1.-4. There is a one-one correspondence between elements $\omega$ of $I_{2}$ and symbol sequences $S(\omega)=\ldots \omega_{2} \omega_{1} \omega_{0}$, where $\omega_{k} \in\{0,1\} . S(\omega)$ with a finite number of 1 is the diadic representation of the nonnegative integer $\omega=\sum \omega_{k} 2^{k}$. A sequence with a finite number of 0 represents a negative number: $S^{-1}(\ldots 111)=-1$ and $S^{-1}\left(\ldots 111 \omega_{n-1} \ldots \omega_{0}\right)=\sum_{k=0}^{n-1} \omega_{k} 2^{k}-2^{n}$. One may use the formal series $\sum_{k=0}^{\infty} \omega_{k} 2^{k}$ as a notation for $\omega$. The order of $\omega \in I_{2}$ is obtained by extension from $\mathbb{Z}$ as $\operatorname{ord}(\omega)$ $=\min \left\{k \mid \omega_{k}=1\right\}$. Arithmetics on $I_{2}$ is extended from $\mathbb{Z}$. If $S(\omega)+S\left(\omega^{\prime}\right), S(\omega) S\left(\omega^{\prime}\right)$ are defined according to the addition and multiplication of integers in diadic representation then $S\left(\omega+\omega^{\prime}\right)=S(\omega)+S\left(\omega^{\prime}\right), S\left(\omega \omega^{\prime}\right)=S(\omega) S\left(\omega^{\prime}\right)$.

Let us return to the potential. It naturally extends to $x \in I_{2}$ via

$$
\frac{1}{\lambda} V(x)=\left\{\begin{array}{lll}
\left(1-R^{\operatorname{ord}(x)}\right) /(1-R), & \text { if } & R \neq 1 \\
\sum_{k=0}^{\operatorname{ord}(x)-1} R^{k}=\operatorname{ord}(x), & \text { if } & R=1 .
\end{array}\right.
$$


Therefore, by the extension to $I_{2}$ of Eq. (2.4),

$$
\frac{1}{\lambda} V(x)=\left\{\begin{array}{lll}
\left(1-|x|_{1 / R}\right) /(1-R), & \text { if } & R<1, \\
-\log _{2}|x|_{2}, & \text { if } & R=1, \\
\left(1-1 /|x|_{R}\right) /(1-R), & \text { if } & R>1, \quad x \neq 0 .
\end{array}\right.
$$

Thus, one may recognize in $V$ a 1-, 2-, and 3-dimensional Coulomb potential for $R<1, R=1$, and $R>1$, respectively. Alternately we can write

$$
\frac{1}{\lambda} V(x)= \begin{cases}\left(1-1 /|x|_{2}^{v}\right) /\left(1-2^{v}\right), & \text { if } \quad v \neq 0 \\ -\log _{2}|x|_{2}, & \text { if } \quad v=0\end{cases}
$$

where $v=\log _{2} R$. Notice the continuity of $V$ in the valuation metric: if $|x-y|_{s}$ $<\min \left\{|x|_{s},|y|_{s}\right\}$, then $V(x)-V(y)=0$. From Eq. (2.5a) and by using Property 4 one easily verifies

$$
V(2 x)=R V(x)+\lambda
$$

This symmetry of dilation is at the origin of the renormalizability of the Schrödinger equation. Let now fix $R<1$. Then $V$ is limit periodic and the set of its translates $V^{(k)}$, where

$$
V^{(k)}(n)=V(n+k),
$$

is precompact in $l_{\infty}$. The closure of $\left\{V^{(k)}\right\}$ with respect to the $l_{\infty}$ norm is the hull of $V$ (see [12]).

Proposition 1. The hull of $V$ is given as

$$
\operatorname{cl}\left(\left\{V^{(k)}\right\}_{k \in \mathbb{Z}}\right)=\left\{V^{(\omega)}\right\}_{\omega \in I_{2}},
$$

where cl stands for closure and

$$
V^{(\omega)}(n)=V(n+\omega)=\lambda\left(1-|n+\omega|_{1 / R}\right) /(1-R) .
$$

Notice that for any $n, V^{(\omega)}(n)$ can be calculated by a finite algorithm, thanks to Property 3.

Proof. Fix any $\omega \in I_{2}$ and choose a sequence of integers $k_{i}$ converging to $\omega$ in $I_{2}:\left|\omega-k_{i}\right|_{s} \rightarrow 0$ as $i \rightarrow \infty$. By the triangle inequality, for any $n \in \mathbb{Z}$

$$
|| n+\left.\omega\right|_{s}-\left|n+k_{i}\right|_{s}|\leqq| \omega-\left.k_{i}\right|_{s} .
$$

Thus, with $s=1 / R$ we find

$$
\left\|V^{(\omega)}-V^{\left(k_{i}\right)}\right\|_{\infty} \leqq \frac{|\lambda|}{1-R}\left|\omega-k_{i}\right|_{1 / R} \rightarrow 0 \quad \text { as } \quad i \rightarrow \infty,
$$

so that $V^{(\omega)}$ is in the hull.

Suppose now that $W$ is in the hull, then there is a sequence of integers $k_{i}$ such that

$$
W(n)=\lim _{i \rightarrow \infty} V\left(n+k_{i}\right)
$$


where the limit exists and is uniform in $n$. The sequence $\left\{k_{i}\right\}$ has at least one accumulation point $\omega$ in $I_{2}$, so that

$$
W(n)=V(n+\omega), \quad \text { all } \quad n \in \mathbb{Z}
$$

or equivalently

$$
W=V^{(\omega)} .
$$

More is true: the above equality holds with a unique $\omega \in I_{2}$. Indeed, assume there exist $\omega, \omega^{\prime} \in I_{2}$ such that

$$
|n+\omega|_{s}=\left|n+\omega^{\prime}\right|_{s}, \quad \text { all } \quad n \in \mathbb{Z} .
$$

Then by Property 3,

$$
\left|\omega-\omega^{\prime}\right|_{s}=\left|n+\omega-\left(n+\omega^{\prime}\right)\right|_{s} \leqq|n+\omega|_{s}, \quad \text { all } n \in \mathbb{Z} .
$$

We can choose a sequence of integers $n_{j}$ such that $n_{j} \rightarrow-\omega$ in $I_{2}$, i.e. $\left|n_{j}+\omega\right|_{s} \rightarrow 0$ proving $\omega=\omega^{\prime}$.

$I_{2}$ with the operation + forms a compact topological group. It carries a unique + invariant probability measure $\mu$, called the Haar measure. $\left(I_{2}, \mu,+\right)$ is a dynamical system with the "dynamics" $\omega \mapsto \omega+1$.

Lemma 1. The Haar measure on the topological group of 2-adic integers is

$$
d \mu(\omega)={\underset{x=0}{X}}_{k}^{\infty} d P\left(\omega_{k}\right)
$$

with $P(0)=P(1)=\frac{1}{2} .\left(I_{2}, \mu,+\right)$ is an ergodic dynamical system.

Proof. Equation (2.6) defines a probability measure on $I_{2}$. Consider the cylinder sets

$$
\Omega_{n}\left(\omega_{n}\right)=\left\{x \in I_{2} \mid x_{n}=\omega_{n}\right\}, \quad \omega_{n} \in\{0,1\}
$$

and for $\omega \in I_{2}$,

$$
B(\omega, n)=\Omega_{0}\left(\omega_{0}\right) \cap \ldots \cap \Omega_{n-1}\left(\omega_{n-1}\right)
$$

Clearly

$$
\mu(B(\omega, n))=\prod_{i=0}^{n-1} P\left(\omega_{i}\right)=1 / 2^{n} .
$$

For $A \subset I_{2}$ let $A+1=\{\omega+1 \mid \omega \in A\}$. Then

$$
\mu(B(\omega, n)+1)=\mu(B(\omega+1, n))=1 / 2^{n} .
$$

Since the cylinders (2.7) form a basis of open sets for the topology on $I_{2}$, the invariance of $\mu$ is proved. It is easy to see that the sets defined in (2.7) are the balls of radius $s^{-n}$ in the metric $|\cdot|_{s}$ :

$$
B(\omega, n)=\left\{x \in I_{2}|| x-\left.\omega\right|_{s} \leqq s^{-n}\right\} .
$$

Balls in $I_{2}$ have several remarkable properties (cf. [20, Chap. 7]):

(i) If $x \in B(\omega, n)$ then $B(x, n)=B(\omega, n)$.

(ii) $B(\omega, n)$ is open and closed, its boundary is empty. 
(iii) $B(\omega, n)$ is disconnected:

$$
B(\omega, n)=B(\omega, n+1) \cup B\left(\omega+2^{n}, n+1\right) .
$$

(iv) The set of all balls is

and

$$
\left\{B(k, n) \mid n \geqq 0, k=0, \ldots, 2^{n}-1\right\},
$$

$$
I_{2}=\bigcup_{k=0}^{2^{n}-1} B(k, n), \quad \text { any } \quad n \geqq 0
$$

(v) For any $\omega \in I_{2}$,

$$
\left\{\omega, \omega+1, \ldots, \omega+2^{n}-1\right\} \cap B(k, n)
$$

contains a unique point for any $k \in\left\{0, \ldots, 2^{n}-1\right\}$.

All these properties follow immediately from Eq. (2.7). Let

Then

$$
m=\sum_{k=0}^{n-1} \omega_{k} 2^{k}
$$

$$
\omega+i \in B\left((m+i)\left(\bmod 2^{n}\right), n\right),
$$

and for the characteristic function $\chi_{B(k, n)}$ one obtains

$$
\lim _{N \rightarrow \infty} \frac{1}{N} \sum_{i=1}^{N} \chi_{B(k, n)}(\omega+i)=1 / 2^{n}=\int \chi_{B(k, n)}(x) d \mu(x)
$$

independently of $\omega$. Ergodicity derives from (2.10) by standard approximation arguments.

Since

$$
V^{(\omega)}(n)=V(\omega+n),
$$

where $\{\omega+n\}_{n \in \mathbb{Z}}$ is a trajectory in an ergodic system, $V^{(\omega)}$ is by definition an ergodic potential. We observe that $V$ is unbounded for $R \geqq 1$ but is $\mu$-integrable for $R<2$, i.e. the mean value of the potential exists for $R<2$. This will be used in Sect. 8 .

\section{Renormalization Group Transformation. The Trace Equation}

Any one-dimensional linear homogeneous second-order difference equation

$$
\psi(n+1)=\alpha_{0}(n) \psi(n)-\beta_{0}(n-1) \psi(n-1)
$$

is exactly renormalizable in the following sense. One can express $\psi(2 n+2)$ in terms of $\psi(2 n)$ and $\psi(2 n-2)$ according to the scheme

Fig. 1

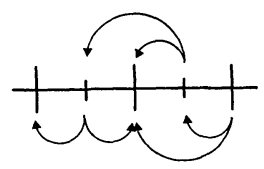


so that the form of the related equation remains unchanged. Performing this "decimation" $m$ times one arrives at the equation

$$
\psi\left((n+1) 2^{m}\right)=\alpha_{m}(n) \psi\left(n 2^{m}\right)-\beta_{m}(n-1) \psi\left((n-1) 2^{m}\right),
$$

where the coefficients are determined via the recursion relation

$$
\begin{aligned}
\alpha_{m+1}(n)= & \alpha_{m}(2 n+1) \alpha_{m}(2 n)-\beta_{m}(2 n) \\
& -\alpha_{m}(2 n+1) \beta_{m}(2 n-1) / \alpha_{m}(2 n-1), \\
\beta_{m+1}(n)= & \alpha_{m}(2 n+3) \beta_{m}(2 n+1) \beta_{m}(2 n) / \alpha_{m}(2 n+1) .
\end{aligned}
$$

Equations (3.3) simplify considerably if the starting point [Eq. (3.1)] is the Schrödinger equation

$$
\psi(n+1)=(E-V(n)) \psi(n)-\psi(n-1)
$$

with the ultrametric potential (1.1). Indeed, choosing

$$
\begin{aligned}
& \beta_{0}(n) \equiv 1, \\
& \alpha_{0}(n)=\alpha_{0, \operatorname{ord}(n)},
\end{aligned}
$$

induction shows that for any $m, \beta_{m}(n) \equiv 1$ and $\alpha_{m}(n)$ depends only on ord $(n)$. With the notation

$$
\alpha_{m}(n)=\alpha_{m, \operatorname{ord}(n)}
$$

and using ord $(2 n)=\operatorname{ord}(n)+1$, Eqs. (3.3) lead to

$$
\alpha_{m+1}(n)=\alpha_{m, 0} \alpha_{m}(2 n)-2
$$

or

$$
\alpha_{m+1, i}=\alpha_{m, 0} \alpha_{m, i+1}-2 .
$$

The initial condition for (3.6) is

$$
\alpha_{0}(n)=E-V(n) .
$$

Equation (3.6) was written down first by Livi et al. [11]. These authors reduced the problem of the solution of Eq. (3.6) to the study of a recursion relation for the sequence $\left\{\alpha_{m, 0}\right\}$ only. Here we give a different derivation of this relation. As a first step we show that

$$
\alpha_{m, 0}=\tau_{m},
$$

where

$$
\tau_{m}=\operatorname{tr} M_{m}=\operatorname{tr}\left(A_{2^{m}} \ldots A_{1}\right)
$$

and

$$
A_{n}=\left(\begin{array}{cr}
E-V(n) & -1 \\
1 & 0
\end{array}\right)
$$


is the transfer matrix at site $n$.

For the potential (1.1), Eq. (3.2) reads as

$$
\psi\left((n+1) 2^{m}\right)=\alpha_{m}(n) \psi\left(n 2^{m}\right)-\psi\left((n-1) 2^{m}\right) .
$$

Setting here $n=1$ we get

$$
\psi\left(2^{m+1}\right)=\alpha_{m, 0} \psi\left(2^{m}\right)-\psi(0)
$$

Another expression can be obtained by solving the Schrödinger equation with the use of transfer matrices. Introduce the vector

then

$$
\Psi_{N}=\left(\begin{array}{c}
\psi(N+1) \\
\psi(N)
\end{array}\right)
$$

$$
\Psi_{N}=A_{N} \ldots A_{1} \Psi_{0} .
$$

Observe that Eq. (2.1) for the potential implies

$$
\begin{aligned}
M_{m+1} & =A_{2^{m+1}} \ldots A_{1}=A_{2^{m+1}} A_{2^{m}}^{-1} M_{m}^{2} \\
& =\left(\begin{array}{cc}
1 & V\left(2^{m}\right)-V\left(2^{m+1}\right) \\
0 & 1
\end{array}\right) M_{m}^{2} .
\end{aligned}
$$

If one applies the characteristic equation of $M_{m}$,

$$
M_{m}^{2}-\tau_{m} M_{m}+\mathbb{1}=0
$$

to the vector $\Psi_{0}$ and accounts for (3.14-15), one obtains

or

$$
\left(\begin{array}{cc}
1 & V\left(2^{m+1}\right)-V\left(2^{m}\right) \\
0 & 1
\end{array}\right) \Psi_{2^{m+1}}-\tau_{m} \Psi_{2^{m}}+\Psi_{0}=0
$$

$$
\psi\left(2^{m+1}+1\right)+\lambda(f(m+1)-f(m)) \psi\left(2^{m+1}\right)-\tau_{m} \psi\left(2^{m}+1\right)+\psi(1)=0,
$$

and

$$
\psi\left(2^{m+1}\right)-\tau_{m} \psi\left(2^{m}\right)+\psi(0)=0 .
$$

$\alpha_{m, 0}=\tau_{m}$ follows from the comparison of (3.12) with Eq. (3.18). It happens that with the choice (1.2) for $f, \tau_{m}$ satisfies an autonomous difference equation (cf. [10]).

Proposition 2. Let $f(m)=\sum_{k=0}^{m-1} R^{k}$. Then

$$
\tau_{m+1}=\tau_{m}^{2}-2+R \tau_{m}\left(\tau_{m}-\tau_{m-1}^{2}+2\right), \quad m \geqq 1 .
$$

Proof. Take the trace of Eq. (3.15) and use

then

$$
\begin{gathered}
\operatorname{tr}\left(M_{m}^{2}\right)=\tau_{m}^{2}-2 \\
\tau_{m+1}=\tau_{m}^{2}-2+\operatorname{tr}\left[\left(\begin{array}{cc}
0 & -\lambda R^{m} \\
0 & 0
\end{array}\right) M_{m}^{2}\right] \\
=\tau_{m}^{2}-2-\lambda R^{m}\left(M_{m}^{2}\right)_{21} \\
=\tau_{m}^{2}-2-\lambda R^{m} \tau_{m}\left(M_{m}\right)_{21}
\end{gathered}
$$


On the other hand, from (3.15)

$$
\left(M_{m}\right)_{21}=\left(M_{m-1}^{2}\right)_{21}=\tau_{m-1}\left(M_{m-1}\right)_{21}=\frac{1}{\lambda R^{m-1}}\left(\tau_{m-1}^{2}-2-\tau_{m}\right),
$$

where in the last equality we applied (3.20) with $m-1$ replacing $m$. Equation (3.20) together with (3.21) gives (3.19).

Remarks. 1. If we suppose only Eq.(1.1), we obtain a formula similar to (3.19) but $R$ replaced by $(f(m+1)-f(m)) /(f(m)-f(m-1))$ which generally depends on $m$. This makes Eq. (3.19) non-autonomous and introduces unnecessary complications in the further discussion. Therefore we fix $\Delta f(m) / \Delta f(m-1)=$ const $=R$ and solve this equation for $f$. The general solution is $f(m)=a+b R^{m}$ which gives (1.2) with $a=-b$ $=1 /(1-R)$.

2. Another useful form is obtained by breaking the iteration (3.19) into two steps. Introducing

$$
\lambda_{m}=\lambda R^{m}\left(M_{m}\right)_{21}
$$

Eqs. (3.20) and (3.21) read as

$$
\tau_{m+1}=\tau_{m}^{2}-2-\lambda_{m} \tau_{m}, \quad \lambda_{m}=R \lambda_{m-1} \tau_{m-1} .
$$

This two-dimensional recurrence was studied in [11].

3. The initial conditions for (3.19) are

$$
\tau_{0}=\operatorname{tr} A_{1}=E, \quad \tau_{1}=\operatorname{tr} A_{2} A_{1}=E(E-\lambda)-2=\tau_{0}^{2}-2-\lambda \tau_{0} .
$$

Comparison with (3.23) shows that $\lambda_{0}=\lambda$ and thus

$$
\lambda_{m}=\lambda R^{m} \tau_{m-1} \ldots \tau_{0}
$$

4. Let $\psi^{0}$ and $\psi^{1}$ be the solutions of $H \psi=E \psi$ with initial conditions

$$
\begin{array}{ll}
\psi^{0}(0)=0, & \psi^{0}(1)=1, \\
\psi^{1}(0)=1, & \psi^{1}(1)=0 .
\end{array}
$$

Then

$$
M_{m}=\left(\begin{array}{cc}
\psi^{0}\left(2^{m}+1\right) & \psi^{1}\left(2^{m}+1\right) \\
\psi^{0}\left(2^{m}\right) & \psi^{1}\left(2^{m}\right)
\end{array}\right)
$$

so that $\left(M_{m}\right)_{21}=\psi^{0}\left(2^{m}\right)$. From (3.22) and (3.25) we get

$$
\lambda_{m}=\lambda R^{m} \psi^{0}\left(2^{m}\right)
$$

and

$$
\psi^{0}\left(2^{m}\right)=\tau_{m-1} \ldots \tau_{0} .
$$

The last equation can be obtained directly from Eq. (3.18). From

Let us return to Eq. (3.6). It can be solved easily under the assumption (1.2).

$$
\alpha_{m, 0}=\tau_{m}, \quad \alpha_{m, 1}=\tau_{m}-\lambda_{m}, \quad \text { and } \quad \alpha_{m, 2}=\tau_{m}-\lambda_{m}(1+R)
$$


we make the guess

$$
\alpha_{m, i}=\tau_{m}-\lambda_{m}\left(1+R+\ldots+R^{i-1}\right)=\tau_{m}-\lambda_{m} f(i)
$$

and verify it by replacing (3.30) into Eq. (3.6b). As a consequence,

$$
\alpha_{m}(n)=\tau_{m}-\frac{\lambda_{m}}{\lambda} V(n)
$$

This formula is valid also for $n=0$ provided that $V(0)=\lambda \sum_{m=0}^{\infty} R^{k}$. Later on, we will need a general formula for $\alpha_{m}(0)$, showing its dependence on $V(0)$. Such a formula is easily obtained from Eq. (3.6a). Set $n=0$, iterate the equation until descending at $\alpha_{0}(0)=E-V(0)$ and use Eqs. (3.8) and (3.29). This gives

$$
\alpha_{m}(0)=\psi^{0}\left(2^{m}\right)\left[E-V(0)-\sum_{k=1}^{m} 2 / \psi^{0}\left(2^{k}\right)\right] .
$$

We have a particular interest in $\alpha_{m}(0)$. The potential is even, $V(n)=V(-n)$, so that any solution of the Schrödinger equation can be written in the form $\alpha \psi^{0}+b \psi^{e}$. Here $\psi^{0}$ is the odd solution introduced in Eq. (3.26) and $\psi^{e}$ is the even solution chosen such that $\psi^{e}(0)=1$. Writing Eq. (3.11) for $\psi^{e}$ and setting $n=0$ we find

$$
\psi^{e}\left(2^{m}\right)=\frac{1}{2} \alpha_{m}(0) \text {. }
$$

Notice that for $R \geqq 1, H \psi=E \psi$ has no even solution because $V(0)=\infty$. A comparison of Eq. (3.31) with Eqs. (3.11) and (3.4) shows that $\tau_{m}$ and $\lambda_{m}$ play the role of the renormalized energy and potential amplitude, respectively. Intuitively one expects that both should be bounded in the continuous spectrum. Our results confirm this for $\tau_{m}$ with the exception of the set $S$ for $R<1$. On the other hand, Eq. (3.25) will imply that $\lambda_{m}$ vanishes for large $m$ in (some) gap-edge states.

\section{Asymptotic Behaviour of the Sequence of Traces}

In this section we classify the sequences $\left\{\tau_{n}\right\}$ which solve Eq. (3.19) according to their asymptotic behaviour. The classification is similar to the one valid for the Fibonacci Hamiltonian [17] with some additional difficulties due essentially to the fact that bounded sequences are not uniformly bounded or at least we cannot prove it.

Our main concern is to describe the unbounded sequences. As to the bounded sequences, we will make use only of the following simple result.

Lemma 2. Let $\tau_{n}=0$. Then $\tau_{n+1}=-2$ and $\tau_{n+k}=2$ for $k>1$.

Proof. This comes immediately from Eq. (3.19).

We say that a sequence $\left\{a_{n}\right\}$ tends monotonically to $A$ if it is monotone for $n$ sufficiently large, and $a_{n} \rightarrow A$.

Definition 1. A solution $\left\{\tau_{n}\right\}$ of Eq. (3.19) is $U_{1}$-type if there exists some $N \geqq 1$ such that

$$
\tau_{N}>\tau_{N-1}^{2}-2 \text { and } \tau_{N}>2
$$


Lemma 3. If $\left\{\tau_{n}\right\}$ is $U_{1}$-type then $\tau_{n}$ tends monotonically to $+\infty$.

Proof. Suppose that (4.1) holds true. Then Eq. (3.19) shows that

$$
\tau_{n+1}>\tau_{n}^{2}-2>\tau_{n}+\left(\tau_{n}-2\right)
$$

for any $n \geqq N$.

Lemma 4. $\left\{\tau_{n}\right\}$ is $U_{1}$-type in the following cases:

(i) $\tau_{N}<-2$ for some $N \geqq 1$.

(ii) $\left|\tau_{N-1}\right| \leqq 2$ and $\tau_{N}>2$ for some $N \geqq 1$.

Proof. In both cases Eq. (3.19) implies

$$
\tau_{N+1}>\tau_{N}^{2}-2>2 \text {. }
$$

The above list is not exhaustive but we do not need more to prove

Proposition 3. Let $\left\{\tau_{n}\right\}$ be unbounded. Then $\tau_{n}$ tends monotonically to $+\infty$.

Proof. Lemma 3 proves it for $U_{1}$-type sequences. Suppose now that $\left\{\tau_{n}\right\}$ is unbounded but not $U_{1}$. Then

$$
2<\tau_{n} \leqq \tau_{n-1}^{2}-2
$$

for all $n \geqq 1$, as one sees from the definition of $U_{1}$ and from Lemma 4 . One can improve the lower bound for $\tau_{n}$ : Let us notice that Eq. (3.19) can be written in the form

$$
\tau_{n}=\frac{R}{R+1}\left(\tau_{n-1}^{2}-2+\frac{2+\tau_{n+1}}{R \tau_{n}}\right) .
$$

Since the last term in the parenthesis is positive, the inequality

$$
\max \left\{2, \frac{R}{R+1}\left(\tau_{n-1}^{2}-2\right)\right\}<\tau_{n} \leqq \tau_{n-1}^{2}-2
$$

holds equally true for all $n \geqq 1$. Now $\left\{\tau_{n}\right\}$ is unbounded, whence there is some $N \geqq 1$ such that $\tau_{N}>2+2 / R$. Then

$$
\begin{aligned}
\tau_{N+1} & >\frac{R}{R+1}\left(\tau_{N}^{2}-2\right)>\frac{R}{R+1}\left(2 \frac{R+1}{R} \tau_{N}-2\right) \\
& =\tau_{N}+\left(\tau_{N}-\frac{2 R}{R+1}\right)>\tau_{N}+2 \frac{2 R+1}{R(R+1)}>2+\frac{2}{R},
\end{aligned}
$$

therefore the estimation can be repeated for $\tau_{N+k}$, all $k \geqq 1$, which gives the result.

Definition 2. $\left\{\tau_{n}\right\}$ is $U_{2}$-type if there exists some $N \geqq 1$ such that

$$
\tau_{N}>R \tau_{N-1}^{2} \text { and } \tau_{N}>1 / R .
$$

Lemma 5. If $\left\{\tau_{n}\right\}$ is $U_{2}$-type then $\tau_{n}$ tends monotonically to $+\infty$. 
Proof. Suppose (4.4) holds true and define $b_{n}$ by $\tau_{n}=b_{n} / R$. Write Eq. (3.19) in the form

$$
\frac{\tau_{N+1}}{\tau_{N}^{2}}=1+R-\frac{R}{\tau_{N} / \tau_{N-1}^{2}}+\frac{2}{\tau_{N}}\left(R-\frac{1}{\tau_{N}}\right) .
$$

Equations (4.4) and (4.5) imply that

$$
\tau_{N+1}=\frac{b_{N+1}}{R}>R \tau_{N}^{2}=\frac{b_{N}^{2}}{R} .
$$

Since (4.4) holds for $N+1$ replacing $N$, the estimation can be repeated. We find

$$
b_{n}>b_{n-1}^{2}>\ldots>b_{N}^{2^{n-N}}
$$

for all $n>N$. This shows monotonicity and divergence.

We denote by $U_{1}, U_{2}$ the families of sequences of $U_{1}$ - or $U_{2}$-type, respectively. From the proof of Lemmas 3, 5 it is clear that

$$
U_{1} \subset U_{2} \text { for } R<1, \quad U_{2} \subset U_{1} \text { for } R \geqq 1 \text {. }
$$

It will be shown that for $R \geqq 1$ all the unbounded sequences are $U_{1}$-type. For $R<1$, $U_{2}$ probably does not include all the unbounded sequences, whence the

Definition 3. For $R<1,\left\{\tau_{n}\right\}$ is $S$-type if it is unbounded but not $U_{2}$-type.

Notice that for $S$-type sequences (4.3) holds true.

We are going to discuss the fixed points of Eq. (3.19) "at the infinity." We suppose that $\left\{\tau_{n}\right\}$ is unbounded and introduce the variables

$$
x_{n}=\tau_{n} / \tau_{n-1}^{2}, \quad \varepsilon_{n}=\frac{2}{\tau_{n}}\left(R-\frac{1}{\tau_{n}}\right) .
$$

Now $\tau_{n} \neq 0$ for any $n$ (otherwise $\left\{\tau_{n}\right\}$ would be bounded, see Lemma 2), therefore $x_{n}$ and $\varepsilon_{n}$ are well-defined. Moreover, $\varepsilon_{n}$ tends monotonically to zero from above. Let

$$
g(x)=1+R-R / x,
$$

then Eq. (4.5) reads as

$$
x_{n+1}=g\left(x_{n}\right)+\varepsilon_{n} .
$$

One might think that the iteration (4.9) is well-approximated by the "unperturbed" iteration

$$
x_{n+1}^{0}=g\left(x_{n}^{0}\right) .
$$

This will prove to be incorrect but the study of (4.10) is nevertheless useful.

Lemma 6. The iteration (4.10) has two fixed points, $R$ and $1: \min \{1, R\}$ is repulsive and $\max \{1, R\}$ is attractive (Fig. 2).

Proof. The solutions of $g(x)=x$ are $R$ and 1. Since $d^{2} g / d x^{2}<1$ for $x>0, d g / d x>1$ for $x=\min \{1, R\}$ and $<1$ for $x=\max \{1, R\}$.

It is easy to see that the stable fixed point $\max \{1, R\}$ remains unchanged and stable under the perturbation $\varepsilon_{n}$ vanishing at $n \rightarrow \infty$. The unstable fixed point may, however, become attractive from below (case $R<1$ ) or disappear (case $R \geqq 1$ ): 


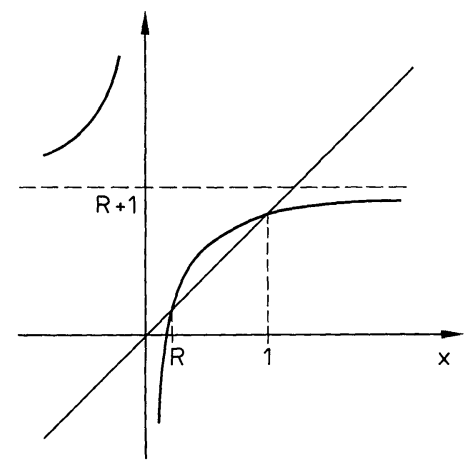

Fig. 2. The function $g(x)$ and the fixed points for $R<1$

Proposition 4. For $\left\{\tau_{n}\right\}$ unbounded the iteration (4.9) is convergent and $x_{n}$ tends either to $R$ or to 1 . For $R \geqq 1$ the only limit point is $R$ and $\left\{\tau_{n}\right\}$ is $U_{1}$-type. For $R<1, x_{n} \rightarrow 1$ corresponds to $\left\{\tau_{n}\right\} \in U_{2}$ and $x_{n} \rightarrow R$ corresponds to an $S$-type $\left\{\tau_{n}\right\}$. In the latter case $x_{n} \uparrow R$.

Proof. Let us consider Eq. (4.9) for $n \geqq N$, where $N$ is chosen so that $\tau_{n}>0$ increases and $\varepsilon_{n}$ decreases monotonically for $n \geqq N$. We distinguish between two cases:

(i) $x_{n} \geqq \min \{1, R\}$. Then due to the facts that $\max \{1, R\}$ is an attractive fixed point of Eq. (4.10), and $\varepsilon_{n} \downarrow 0, x_{n}$ tends to $\max \{1, R\}$.

(ii) $x_{N}<\min \{1, R\}$. Then $x_{n}$ increases monotonically provided that $x_{n}<\max \{1, R\}$. Indeed:

Let us observe at first that $x_{n}>0$ for all $n \geqq N$ and $g^{\prime}(x)>0$ for $x>0$. Suppose that $x_{n+1}<x_{n}$, then $g\left(x_{n+1}\right)<g\left(x_{n}\right)$ and

$$
x_{n+2}=g\left(x_{n+1}\right)+\varepsilon_{n+1}<g\left(x_{n}\right)+\varepsilon_{n}=x_{n+1},
$$

so that $x_{n}$ decreases monotonically. Therefore $x_{n} \rightarrow X<\min \{1, R\} . X=0$ can be excluded, for $x_{n} \rightarrow 0$ would imply $g\left(x_{n}\right) \rightarrow-\infty$ and $x_{n}<0$ for large $n$. Owing to the continuity of $g(x)$ for $x>0, X$ is a solution of $x=g(x)$, therefore $X=1$ or $R$ which contradicts $X<\min \{1, R\}$.

Now there are two possibilities. First, $x_{n}$ may jump above $\min \{1, R\}$ and then tend to $\max \{1, R\}$ according to (i). Second, it may increase monotonically and remain bounded by $\min \{1, R\}$, and then to converge to $X=g(X)=\min \{1, R\}$.

By (i) and (ii) it is proved that $\lim x_{n}$ exists and is either 1 or $R$. Let now $R<1$. Then $x_{n} \rightarrow 1$ implies $x_{n}>R$ for large $n$. This and $\tau_{n} \rightarrow+\infty$ result in (4.4) and $\left\{\tau_{n}\right\} \in U_{2}$. On the other hand, $\left\{\tau_{n}\right\}$ is $S$-type if $x_{n} \rightarrow R$ since the convergence is from below [cf. (ii)].

Let $R>1$. If $x_{n} \rightarrow R$, then $x_{n}>1$ for large $n$. This and $\tau_{n} \rightarrow \infty$ give (4.1) which makes $\left\{\tau_{n}\right\} U_{1}$-type. Below we show that for $R=1, x_{n}$ cannot converge to 1 from below, and for $R>1, x_{n}$ cannot converge to 1 at all. This proves that each unbounded $\left\{\tau_{n}\right\}$ is $U_{1}$-type for $R \geqq 1$ and, in particular, is $U_{2}$-type for $R=1$. In fact, from Eqs. (3.23) and (3.25),

$$
x_{n+1}=1-\frac{2}{\tau_{n}^{2}}-\lambda R^{n} \frac{\tau_{n-1} \ldots \tau_{0}}{\tau_{n}}=1-\frac{2}{\tau_{n}^{2}}-\frac{\lambda R^{n}}{\tau_{0} x_{1} x_{2} \ldots x_{n}} .
$$


This immediately shows that for $R>1, \tau_{n} \rightarrow \infty$ and $x_{n} \rightarrow 1$ are incompatible, and so are $\tau_{n} \rightarrow \infty$ and $x_{n} \uparrow 1$ for $R=1$.

By the above proposition we finished the classification of the solutions of Eq. (3.19) for the purposes of the present paper. For $R<1$, there are three classes: bounded, $S$-, and $U_{2}$-type. For $R \geqq 1$ there are only two classes: bounded and $U_{1}$-type.

\section{Locating the Spectrum}

We want to show that bounded and $S$-type sequences correspond to energies in the spectrum of the operator $H$ while $U$-type $\left(U_{1}\right.$ or $\left.U_{2}\right)$ sequences belong to energies outside the spectrum. The method is similar to that applied in [17].

Let $W=\{W(n)\}_{-\infty}^{\infty}$ be any (bounded or unbounded) real sequence,

$$
\begin{aligned}
A & =H_{0}+W, \\
\left(H_{0} \psi\right)(n) & =\psi(n+1)+\psi(n-1), \quad(W \psi)(n)=W(n) \psi(n),
\end{aligned}
$$

and let $\sigma(A)$ denote the spectrum of $A$ and $\sigma_{p p}(A)$ the set of eigenvalues of $A$.

Lemma 7. Suppose that for any nonzero solution of $A \psi=E \psi$,

$$
\sum_{n=0}^{\infty}|\psi(n)|^{2}=\infty
$$

Then $E \in \sigma(A) \backslash \sigma_{p p}(A)$.

Proof. Let

$$
\delta_{k}(n)= \begin{cases}1, & n=k \\ 0, & n \neq k .\end{cases}
$$

Suppose that $E$ is outside the spectrum, then both

$$
(A-E) \phi=\delta_{0} \quad \text { and } \quad(A-E) \phi=\delta_{1}
$$

have a square-summable solution which we denote by $\phi_{0}$ and $\phi_{1}$, respectively. Equation (5.2) is equivalent to

$$
\phi_{k}(n+1)+\phi_{k}(n-1)+(W(n)-E) \phi_{k}(n)= \begin{cases}0, & n \neq k \\ 1, & n=k\end{cases}
$$

for $k=0,1 . \phi_{k} \in l_{2}(\mathbb{Z})$ and Eq. (5.1) imply

$$
\begin{array}{ll}
\phi_{k}(n)=0, & n \geqq k, \\
\phi_{k}(n) \rightarrow 0, & n \rightarrow-\infty .
\end{array}
$$

Since for $n<0$ both $\phi_{0}$ and $\phi_{1}$ satisfy the homogeneous equation, the Wronskian

$$
W\left[\phi_{1}, \phi_{0}\right]=\phi_{1}(n+1) \phi_{0}(n)-\phi_{1}(n) \phi_{0}(n+1)
$$

is constant for $n<0$. However, calculating $W\left[\phi_{1}, \phi_{0}\right]$ at $n=-1$ we get 1 while at $n \rightarrow-\infty$ we get 0 . Therefore $E \in \sigma(A)$ and due to (5.1) it is not an eigenvalue. 
In the previous section we considered $\left\{\tau_{n}\right\}$ independently of its original definition $\tau_{n}=\operatorname{tr} M_{n}$. Henceforward we retake this definition, which means that $\left\{\tau_{n}\right\}$ is understood to be the solution of Eq. (3.19) with the initial conditions (3.24). Thus, $\tau_{n}$ is a $2^{n}$ th degree polynomial of $E$. Let us introduce the following notations:

$$
\begin{aligned}
& B=\left\{E \in \mathbb{R} \mid\left\{\tau_{n}\right\} \text { is bounded }\right\}, \\
& S=\left\{E \in \mathbb{R} \mid\left\{\tau_{n}\right\} \text { is } S \text {-type }\right\}, \\
& U=\left\{E \in \mathbb{R} \mid\left\{\tau_{n}\right\} \text { is } U \text {-type }\right\} .
\end{aligned}
$$

According to Proposition 4,

$$
\mathbb{R}=\left\{\begin{array}{lll}
B \cup S \cup U & \text { if } & R<1, \\
B \cup U & \text { if } & R \geqq 1,
\end{array}\right.
$$

and neither of these sets depend on $V(0)$.

Proposition 5. $B \subset \sigma(H) \backslash \sigma_{p p}(H)$. The set $B$ is nonempty and is dense in $\sigma(H)$.

Proof. Assume that $\left\{\tau_{n}\right\}$ is bounded.

(i) $R<1$. Let us take the norm of Eq. (3.16). $V\left(2^{m+1}\right)-V\left(2^{m}\right)=\lambda R^{m}$, so that

$$
\begin{aligned}
\left\|\Psi_{0}\right\| & \leqq\left\|\left(\begin{array}{cc}
1 & \lambda R^{m} \\
0 & 1
\end{array}\right)\right\| \cdot\left\|\Psi_{2^{m+1}}\right\|+\left|\tau_{m}\right| \cdot\left\|\Psi_{2^{m}}\right\| \\
& \leqq c\left(\left\|\Psi_{2^{m+1}}\right\|+\left\|\Psi_{2^{m}}\right\|\right)
\end{aligned}
$$

with some suitably chosen $c>1$. Therefore

$$
\max \left\{\left\|\Psi_{2^{m+1}}\right\|,\left\|\Psi_{2^{m}}\right\|\right\} \geqq \frac{1}{2 c}\left\|\Psi_{0}\right\|>0
$$

for any $m \geqq 0, \psi(n) \nrightarrow 0$ as $n \rightarrow \infty$ and Lemma 7 applies: $E \in \sigma(H) \backslash \sigma_{p p}(H)$.

(ii) $R \geqq 1$. Let $\psi(0) \neq 0$. From Eq. (3.18),

$$
\psi\left(2^{m+1}\right)-\tau_{m} \psi\left(2^{m}\right)=-\psi(0)
$$

all $m \geqq 0$. Let now $\psi(0)=0$, then $\psi(1) \neq 0$. Take at first $R=1$, then Eq. (3.17) reads for all $m \geqq 0$ as

$$
\psi\left(2^{m+1}+1\right)+\lambda \psi\left(2^{m+1}\right)-\tau_{m} \psi\left(2^{m}+1\right)=-\psi(1)
$$

Take $R>1$. If

$$
\lambda R^{m} \psi\left(2^{m+1}\right) \nrightarrow-\psi(1)
$$

then $\psi(n) \nrightarrow 0$ as $n \rightarrow \infty$, as it is seen immediately from Eq. (3.17). Suppose therefore

Then

$$
\psi\left(2^{m+1}\right)=-\frac{\psi(1)}{\lambda R^{m}}+o\left(R^{-m}\right) \quad \text { as } \quad m \rightarrow \infty .
$$

$$
\begin{aligned}
\psi\left(2^{m+1}+1\right)+\psi\left(2^{m+1}-1\right) & =\left(E-\lambda \frac{R^{m+1}-1}{R-1}\right)\left(-\frac{\psi(1)}{\lambda R^{m}}+o\left(R^{-m}\right)\right) \\
& =\frac{R \psi(1)}{R-1}+o(1) \text { as } m \rightarrow \infty
\end{aligned}
$$


All the Eqs. (5.5) $-(5.8)$ imply that $\psi(n) \nrightarrow 0$ as $n \rightarrow \infty$. Therefore Lemma 7 applies, $E \in \sigma(H) \backslash \sigma_{p p}(H)$.

Now from the argument above and from Lemma 2 it follows that the zeros of the polynomials $\tau_{n}(E)$ are in the spectrum. By Eq. (3.29), these are also the zeros of the polynomials $\psi^{0}\left(2^{n}\right)(E)$. For $R<1$, when the spectrum is bounded, a theorem on orthogonal polynomials [21, Theorem 6.1.1] assures that these zeros are dense in the spectrum. We anticipate and prove in the next section that this holds also for $R \geqq 1$.

Proposition 6. Let $R<1$. Then $S \subset \sigma(H)=\sigma_{\text {ess }}\left(H^{\prime}\right)$ and the even solution of $H \psi=E \psi$ for $E \in S$ decays exponentially at least on the subset $\left\{2^{m}\right\}_{m=0}^{\infty}$. For $E \in S, H^{\prime} \psi=E \psi$ has no polynomially bounded solution.

We interpose

Lemma 8. For $E \in S$, the limit of $E-\sum_{k=1}^{m} 2 / \psi^{0}\left(2^{k}\right)$ exists and is independent of $E$ :

$$
E-\sum_{k=1}^{\infty} \frac{2}{\psi^{0}\left(2^{k}\right)}=\frac{\lambda}{1-R} .
$$

Proof. $\psi^{0}\left(2^{k}\right)$ is independent of $V(0)$ for any $k$, see Eqs. (3.9) and (3.29). Fix $V(0)=\lambda /(1-R)$. Then Eq. (3.31) is valid for $n=0$,

$$
\alpha_{m}(0)=\tau_{m}-\frac{\lambda_{m}}{1-R} .
$$

Let $\left\{\tau_{m}\right\}$ be an $S$-type sequence. From Proposition 4 and Eq. (3.23),

$$
x_{m+1}=\frac{\tau_{m+1}}{\tau_{m}^{2}}=1-\frac{2}{\tau_{m}^{2}}-\frac{\lambda_{m}}{\tau_{m}} \rightarrow R
$$

or

$$
\frac{\lambda_{m}}{\tau_{m}} \rightarrow 1-R
$$

Comparing this with Eq. (5.10), we see that

$$
\frac{\alpha_{m}(0)}{\tau_{m}}=1-\frac{\lambda_{m} / \tau_{m}}{1-R} \rightarrow 0
$$

On the other hand,

$$
\frac{\tau_{m}}{\psi^{0}\left(2^{m}\right)}=\frac{\tau_{m}}{\tau_{m-1} \ldots \tau_{0}}=x_{m} \ldots x_{1} \tau_{0}<c R^{m}
$$

with a suitable finite $c$, since $x_{m} \uparrow R$. Equations (5.11) and (5.12) together with Eq. (3.32) give

$$
\lim _{m \rightarrow \infty} \frac{\alpha_{m}(0)}{\psi^{0}\left(2^{m}\right)}=E-\frac{\lambda}{1-R}-\lim _{m \rightarrow \infty} \sum_{k=1}^{m} \frac{2}{\psi^{0}\left(2^{k}\right)}=0
$$


Proof of Proposition 6. For $E \in S$ we may write

$$
\psi^{e}\left(2^{m}\right)=\frac{1}{2} \psi^{0}\left(2^{m}\right)\left[\frac{\lambda}{1-R}-V(0)+\sum_{k=m+1}^{\infty} 2 / \psi^{0}\left(2^{k}\right)\right],
$$

see Eqs. (3.32), (3.33) and the preceding lemma.

$\tau_{m} / \tau_{m-1}^{2}$ tends monotonically to $R$ from below, thus for any $K<R$ there exists $N$ such that

$$
\tau_{m}>K \tau_{m-1}^{2} \text { if } m>N
$$

Iterating this inequality we find

$$
\tau_{m}>\frac{1}{K}\left(K \tau_{m_{0}}\right)^{2^{m-m_{0}}}
$$

for any $m_{0} \geqq N$ and any $m>m_{0}$. Since $\tau_{m}$ tends to $+\infty$ (Proposition 3$), m_{0}$ can be chosen so that $K \tau_{m_{0}}>1$. From (5.12) and (5.14)

$$
\psi^{0}\left(2^{m}\right)>\tau_{m}>\frac{1}{K}\left(K \tau_{m_{0}}\right)^{2^{m-m_{0}}},
$$

that is, $\psi^{0}(n)$ grows exponentially.

Fix now $V(0)=\lambda /(1-R)$. Then (5.13) and (3.29) yield

$$
\tau_{m} \psi^{e}\left(2^{m}\right)=1+1 / \tau_{m+1}+1 / \tau_{m+1} \tau_{m+2}+\ldots \rightarrow 1,
$$

i.e. $\psi^{e}$ decays exponentially on the sites $2^{m}$. In particular,

$$
\psi^{e}\left(2^{m}\right) / \psi^{0}\left(2^{m}\right) \rightarrow 0, \quad m \rightarrow \infty .
$$

There are two possibilities. If $\psi^{e}$ is square summable then $E$ is an eigenvalue of the operator $H$. If $\psi^{e}$ is not square summable then by (5.15) and (5.16) the condition (5.1) holds true for any solution of $H \psi=E \psi$. In this case Lemma 7 applies, $E \in \sigma(H) \backslash \sigma_{p p}(H)$. In both cases, $E \in \sigma_{\text {ess }}(H)$. Indeed, the potential is ergodic and limit periodic so that $\sigma\left(H_{0}+V^{(\omega)}\right)=\sigma(H)$ for all $\omega \in I_{2}$ and by Pastur's theorem [22] $\sigma(H)=\sigma_{\text {ess }}(H)$.

If $V(0) \neq \lambda /(1-R)$ then $S \subset \sigma_{\text {ess }}\left(H^{\prime}\right)$ remains valid, however $(5.16)$ will be replaced by

$$
\psi^{e}\left(2^{m}\right) / \psi^{0}\left(2^{m}\right) \rightarrow \frac{1}{2}\left[\frac{\lambda}{1-R}-V(0)\right] \neq 0
$$

as $m \rightarrow \infty$. Any solution of $H^{\prime} \psi=E \psi$ can be written as

$$
\psi=a \psi^{0}+b \psi^{e},
$$

therefore any solution grows exponentially at least at one side.

Notice that we did not prove that $S$ is nonempty. $S=\emptyset$ may eventually happen to be true.

We define periodic approximations to $H$ by

$$
H_{n}=H_{0}+V_{n},
$$

where $V_{n}$ is the periodic potential determined by Eq. (2.2). As it is well-known,

$$
\sigma\left(H_{n}\right)=\left\{E \in \mathbb{R}|| \tau_{n} \mid \leqq 2\right\}
$$


since $M_{n}$ is the transfer matrix associated with the periodic Schrödinger equation $H_{n} \psi=E \psi$.

Proposition 7.

$$
S \cap \bigcup_{n=0}^{\infty} \sigma\left(H_{n}\right)=\emptyset
$$

Proof. If $\left\{\tau_{n}\right\}$ is an $S$-type sequence then it satisfies (4.3), so that

$$
S \cap \sigma\left(H_{n}\right)=\emptyset \text { for } n \geqq 1 \text {. }
$$

$S \cap \sigma\left(H_{0}\right)=\emptyset$ is equally true, for $\left|\tau_{0}\right| \leqq 2$ and $\left\{\tau_{n}\right\}$ unbounded implies that $\left\{\tau_{n}\right\}$ is $U_{1}$-type (cf. Lemma 4).

Numerical calculations (Fig. 3a) seem to suggest that for $R<1$ the set $\bigcup_{n=0}^{\infty} \sigma\left(H_{n}\right)$ covers the whole spectrum $\sigma(H)$ with the exception of the upper $(\lambda>0)$ or the lower $(\lambda<0)$ boundary, to which $S$ would then be reduced. In any case, $S$ is separated from the lower $(\lambda>0)$ or from the upper $(\lambda<0)$ part of the spectrum: $V_{0} \equiv 0$, therefore

$$
S \cap \sigma\left(H_{0}\right)=S \cap[-2,2]=\emptyset,
$$

while inf $\sigma(H)>-2$ if $\lambda>0$, and $\sup \sigma(H)<2$ if $\lambda<0$.

For a self-adjoint operator $A$ let

$$
\varrho(A)=\mathbb{R} \backslash \sigma(A) .
$$

Lemma 9. For $R<1$

$$
\varrho(H)=\bigcup_{N}\left(\bigcap_{n \geqq N} \varrho\left(H_{n}\right)\right)^{\mathrm{int}}=\lim _{N \rightarrow \infty}\left(\bigcap_{n \geqq N} \varrho\left(H_{n}\right)\right)^{\mathrm{int}},
$$

where $\wedge$ int means the interior of $\wedge$.

Proof. For $R<1,\left\{H_{n}\right\}$ and $H$ form a family of uniformly bounded self-adjoint operators and, owing to Eq. (2.3), $H_{n}$ converges to $H$ in norm. Thus $H_{n} \rightarrow H$ also in the norm resolvent sense and

$$
\varrho(H) \subset \bigcup_{N}\left(\bigcap_{n \geqq N} \varrho\left(H_{n}\right)\right)^{\mathrm{int}}
$$

is a way of writing what Theorem VIII.23a of Reed-Simon [23] states. The inclusion in the opposite sense follows from Lemma 3 of [17] or from Theorem VIII.24a of [23], since convergence in norm implies strong convergence.

Remark. Apart from the general theorem [22], there is a direct and easy proof that for $R<1, \sigma(H)$ does not contain isolated points. Suppose that $E_{0} \in \sigma(H)$ is isolated. Let $\psi$ be the corresponding normalised eigenvector, $H \psi=E_{0} \psi,\|\psi\|=1$. Then by the functional calculus

$$
\psi(l)^{2}=\frac{1}{2 \pi i} \int_{\Gamma} g_{z}(l) d z
$$

where

$$
g_{z}(l)=\left(\delta_{l},(z-H)^{-1} \delta_{l}\right)
$$


and the integration is done on a contour $\Gamma$ which separates $E_{0}$ from the rest of the spectrum. Using that $H_{n}$ tends to $H$ in the norm resolvent sense, one sees that $g_{z}(l)$ and its integral over $\Gamma$ is limit periodic which contradicts $\psi \in l_{2}$.

If $V(0)$ differs from $\lambda /(1-R)$ then isolated points may enter the spectrum. From Eq. (5.21) we still have

$$
\sigma_{\text {ess }}\left(H^{\prime}\right)=\sigma(H)=\bigcap_{N} \operatorname{cl}\left(\bigcup_{n \geqq N} \sigma\left(H_{n}\right)\right)=\lim _{N \rightarrow \infty} \mathrm{cl}\left(\bigcup_{n \geqq N} \sigma\left(H_{n}\right)\right),
$$

where cl means closure. Notice that the right member of Eqs. (5.21) and (5.23) does not depend on $V(0)$.

The decomposition (5.4) and Propositions 5 and 6 show that for all $R>0$,

$$
\varrho(H) \subset U .
$$

Theorem 1. Let $R<1$. Then $\varrho(H)=U$, i.e.

$$
\sigma(H)=B \cup S=\left\{E \in \mathbb{R} \mid\left\{\tau_{n}\right\} \text { is bounded or }\left\{\tau_{n}\right\} \text { is unbounded and } \tau_{n} / \tau_{n-1}^{2} \rightarrow R\right\} \text {, }
$$

the spectrum contains no isolated point and there is no eigenvalue in the domain

$$
[-2,2] \cup \bigcup_{n=1}^{\infty} \sigma\left(H_{n}\right) .
$$

If $V(0) \neq \lambda /(1-R)$ then $\sigma_{\mathrm{ess}}\left(H^{\prime}\right)$ supports a purely continuous spectral measure class.

Proof. For $R<1$, Eqs. (5.3) and (4.6) yield

$$
U=\left\{E \in \mathbb{R} \mid\left\{\tau_{n}\right\} \text { is } U_{2} \text {-type }\right\} .
$$

Define the open set

$$
O_{N}=\left\{E \in \mathbb{R} \mid \tau_{n}>\max \left\{R \tau_{N-1}^{2}, 1 / R, 2\right\}\right\} .
$$

According to Definition 2, $O_{N} \subset U_{2}$, and from Lemma 5 follows that $O_{N}$ grows monotonically and tends to $U$ which is therefore open. Let $E \in U$, then $E \in O_{N}$ for $N$ sufficiently large. Since $O_{N} \subset \varrho\left(H_{n}\right)$ for all $n \geqq N$,

$$
O_{N} \subset\left(\bigcap_{n \geqq N} \varrho\left(H_{n}\right)\right)^{\text {int }},
$$

where we could take the interior part, $O_{N}$ being open. The limit of this equation as $N \rightarrow \infty$ yields

$$
U \subset \varrho(H),
$$

where we used Lemma 9. This and Eq. (5.24) give $\varrho(H)=U$ which is equivalent to Eq. (5.25). The eigenvalues, if any, belong to $S$. Their absence in the domain (5.26) is a consequence of Proposition 7. If $V(0) \neq \lambda /(1-R)$ then $H^{\prime} \psi=E \psi$ has no $l_{2}$-solution for $E \in B \cup S$, as we have shown in Propositions 5 and 6 . Therefore any spectral measure restricted to

$$
\sigma_{\text {ess }}\left(H^{\prime}\right)=B \cup S
$$

is purely continuous. 
In order to prove an analogous theorem for $R \geqq 1$, we need the counterpart of Lemma 9. Let $H_{n}^{r}$ denote the restriction of $H_{n}$, defined in Eq. (5.17), to $\{\psi(k)\}_{k=1}^{\infty}$ with boundary condition $\psi(0)=0$.

Lemma 10. (i) $\sigma\left(H_{n}^{r}\right)=\sigma\left(H_{n}\right)$.

(ii) For $R \geqq 1, H_{n}^{r}$ tends to $H$ in the strong resolvent sense.

(iii) For any $R \geqq 1$,

$$
\bigcup_{N}\left(\bigcap_{n \geqq N} \varrho\left(H_{n}\right)\right)^{\text {int }} \subset \varrho(H) .
$$

Proof. (i) Let us observe that $V_{n}$ is even: $V_{n}(-k)=V_{n}(k)$. Indeed, from Eqs. (2.1) and (2.2) $V_{n}(-k)=V(-k)=V(k)=V_{n}(k)$ if $k \neq l \cdot 2^{n}, V_{n}\left(-l \cdot 2^{n}\right)=V\left(2^{n}\right)=V_{n}\left(l \cdot 2^{n}\right)$.

Therefore $\sigma_{\text {ess }}\left(H_{n}^{r}\right)=\sigma\left(H_{n}\right)$, the latter being purely continuous. Suppose that $H_{n}^{\mathrm{r}} \phi=E \phi$ has a solution $\phi \in l_{2}(\mathbb{N})$. Then $H_{n} \psi=E \psi$ admits an odd solution such that $\psi(k)=\phi(k)$ for $k>0$, and hence $\psi \in l_{2}(\mathbb{Z})$. This however contradicts the continuity of the spectrum of $H_{n}$. Thus, the spectrum of $H_{n}^{r}$ is also continuous and the two spectra coincide.

(ii) Let $z \in \mathbb{C}, \operatorname{Im} z \neq 0$ and $\psi \in l_{2}(\mathbb{N})$. Then

$$
\begin{aligned}
\left\|\left[(z-H)^{-1}-\left(z-H_{n}^{r}\right)^{-1}\right] \psi\right\| & =\left\|\left(z-H_{n}^{r}\right)^{-1}\left(H-H_{n}^{r}\right)(z-H)^{-1} \psi\right\| \\
& \leqq \frac{1}{|\operatorname{Im} z|}\left\|\left(H-H_{n}^{r}\right) \psi^{\prime}\right\|,
\end{aligned}
$$

where $\psi^{\prime}=(z-H)^{-1} \psi$. Now

$$
\begin{aligned}
\left\|\left(H-H_{n}^{r}\right) \psi^{\prime}\right\|^{2} & =\sum_{k=1}^{\infty}\left[V(k)-V_{n}(k)\right]^{2}\left|\psi^{\prime}(k)\right|^{2} \\
& =\sum_{m=1}^{\infty}\left[V\left(m \cdot 2^{n}\right)-V\left(2^{n}\right)\right]^{2}\left|\psi^{\prime}\left(m \cdot 2^{n}\right)\right|^{2} \\
& \leqq \sum_{m=2}^{\infty}\left|V\left(m \cdot 2^{n}\right) \psi^{\prime}\left(m \cdot 2^{n}\right)\right|^{2} \\
& \leqq \sum_{k=2^{n+1}}^{\infty}\left|V(k) \psi^{\prime}(k)\right|^{2}
\end{aligned}
$$

which goes to zero as $n$ goes to infinity, because $\psi^{\prime} \in D(H)$, the domain of $H$.

(iii) Using (ii) and Theorem VIII.24a of [23] one obtains

$$
\bigcup_{N}\left(\bigcap_{n \geqq N} \varrho\left(H_{n}^{r}\right)\right)^{\text {int }} \subset \varrho(H) .
$$

But $\varrho\left(H_{n}^{r}\right)=\varrho\left(H_{n}\right)$ as we learned from (i), whence (5.27) follows.

Theorem 2. Let $R \geqq 1$. Then $\varrho(H)=U$, i.e.

$$
\sigma(H)=B=\left\{E \in \mathbb{R} \mid\left\{\tau_{n}\right\} \text { is bounded }\right\},
$$

and the spectrum is purely singular continuous.

Proof. If $R \geqq 1$ then $\mathbb{R}=B \cup U$. Since $\varrho(H) \subset U$ [see Eq. (5.24)], it remains to prove that $U \subset \varrho(H)$. 
For $R \geqq 1$ we obtain from Eq. (4.6) and Definition 1 that

$$
U=\left\{E \in \mathbb{R} \mid\left\{\tau_{n}\right\} \text { is } U_{1} \text {-type }\right\}=\bigcup Q_{N}=\lim _{N \rightarrow \infty} Q_{N},
$$

where

$$
Q_{N}=\left\{E \in \mathbb{R} \mid \tau_{N}>\max \left\{\tau_{N-1}^{2}-2,2\right\}\right\} .
$$

The sets $Q_{N}$ are open which shows that $U$ is open. Let $E \in U$, then $E \in Q_{N}$ for $N$ sufficiently large. Since $Q_{N} \subset \varrho\left(H_{n}\right)$ for all $n \geqq N$, we find

$$
Q_{N} \subset\left(\bigcap_{n \geqq N} \varrho\left(H_{n}\right)\right)^{\text {int }} .
$$

Equation (5.27) then implies $Q_{N} C \varrho(H)$ for all $N$, from which $U \subset \varrho(H)$ is obtained as $N$ goes to infinity. $B$ is a closed set and, according to Proposition 5, it supports a purely continuous spectral measure. A recent theorem by Simon and Spencer [26] asserts that $H_{0}+W$ has no absolutely continuous spectrum if both $\{W(n)\}_{n=1}^{\infty}$ and $\{W(-n)\}_{n=1}^{\infty}$ are unbounded. This theorem applies to our case, thus the spectrum is purely singular continuous.

We may summarize what is common in Theorems 1 and 2 as follows.

Corollary of Theorems 1, 2. For any $R>0$,

$$
\sigma(H)=\mathbb{R} \backslash U,
$$

and there is no isolated point in the spectrum.

\section{Cantor Spectrum, IDS and Gap Labelling}

The entire discussion of this section is based on the fact that we know explicitly a dense set in the spectrum: the zeros of the polynomials $\tau_{n}(E)$. We stress that such a knowledge is quite unusual in direct (not inverse) spectral problems exhibiting Cantor spectra.

Let us consider the Dirichlet problem on the internal $\left[1,2^{n}-1\right]$ assigned to $H$. More precisely, let $H_{n}^{D}$ be given by the semi-infinite matrix

$$
\left(H_{n}^{D}\right)_{i j}= \begin{cases}H_{i j}, & \text { if } l \cdot 2^{n}<i, j<(l+1) 2^{n}, \quad l=0,1,2, \ldots, \\ 0, & \text { otherwise }\end{cases}
$$

The solution of $H_{n}^{D}=E \psi$ in the interval $\left[1,2^{n}-1\right]$ corresponds to the solution of $H \psi=E \psi$ with boundary conditions $\psi(0)=\psi\left(2^{n}\right)=0$. Due to the translational symmetry (2.1) of the potential and to the "decoupling" at the sites $l \cdot 2^{n}, H_{n}^{D}$ is the infinite direct sum of the copies of a finite rank operator with $2^{n}-1$ eigenvalues of multiplicity 1 . The spectrum of $H_{n}^{D}$ consists of these $2^{n}-1$ points, each being a simple zero of the polynomial $\psi^{0}\left(2^{n}\right)(E)$ : the condition $\psi(0)=0$ singles out $\psi^{0}$ given by Eq. (3.26).

Let $H^{r}$ denote the restriction of $H$ to $\{\psi(n)\}_{n=1}^{\infty}$ with boundary condition $\psi(0)=0$. By our convention, $H^{r}=H$ for $R \geqq 1$. 
Lemma 11. For all $R>0$, the spectrum of $H^{r}$ is purely continuous and

$$
\sigma\left(H^{r}\right)=\sigma(H) \text {. }
$$

Proof. Any eigenvalue of $H^{r}$ would belong to $U$ or $S$, and $\psi^{0}$ of Eq. (3.26) would be the corresponding eigenvector. However,

$$
\psi^{0}\left(2^{m}\right)=\tau_{m-1} \ldots \tau_{0}
$$

diverges if $E \in U$ or $E \in S$. Therefore $\sigma\left(H^{r}\right)$ is continuous. The potential $V$ is even, hence $\sigma_{\text {ess }}(H)=\sigma_{\text {ess }}\left(H^{r}\right)$. The result follows from the absence of isolated points in both $\sigma(H)$ and $\sigma\left(H^{r}\right)$.

\section{Lemma 12.}

$$
\sigma\left(H_{n}^{D}\right)=\bigcup_{k=0}^{n-1}\left\{E \in \mathbb{R} \mid \tau_{k}(E)=0\right\} .
$$

(ii) For any $R>0, H_{n}^{D}$ tends to $H^{r}$ in the strong resolvent sense.

(iii) For any $R>0$,

$$
\bigcup_{N}\left(\bigcap_{n \geqq N} \varrho\left(H_{n}^{D}\right)\right)^{\text {int }} \subset \varrho(H) .
$$

Proof. Equation (6.1) is an immediate consequence of $\psi^{0}\left(2^{n}\right)=\tau_{n-1} \ldots \tau_{0}$ [see Eq. (3.29)]. The proof of (ii) is analogous to that of (ii) of Lemma 10. One uses

$$
\left(H^{r}-H_{n}^{D}\right)_{i j}= \begin{cases}H_{i j}, & \text { if } i=l \cdot 2^{n} \text { or } j=l \cdot 2^{n}, \\ 0, & \text { otherwise }\end{cases}
$$

and the estimate

$$
\begin{aligned}
\left\|\left(H^{r}-H_{n}^{D}\right) \psi^{\prime}\right\| & =\sum_{l=1}^{\infty}\left[\left|\left(H^{r} \psi^{\prime}\right)\left(l \cdot 2^{n}\right)\right|^{2}+2\left|\psi^{\prime}\left(l \cdot 2^{n}\right)\right|^{2}\right] \\
& \rightarrow 0 \quad \text { as } n \rightarrow \infty,
\end{aligned}
$$

where $\psi^{\prime}=\left(z-H^{r}\right)^{-1} \psi, \operatorname{Im} z \neq 0$ and $\psi \in l_{2}(\mathbb{N})$. Equation (6.2) follows from (ii) via Theorem VIII.24a of [23] and from Lemma 11.

Proposition 9. For any $R>0$ and any $N \geqq 0$, the zeros of the polynomials $\left\{\tau_{n}(E)\right\}_{n \geqq N}$ are dense in the spectrum of $H$.

Proof. According to Eq. (6.1) the claim is

$$
\operatorname{cl}\left(\bigcup_{n \geqq N} \sigma\left(H_{n}^{D}\right)\right)=\sigma(H) .
$$

Now $\sigma\left(H_{n}^{D}\right) \subset \sigma(H)$ for all $n$, see Proposition 5. The spectrum is closed, thus $C$ holds in Eq. (6.3), and $\supset$ follows by complementing Eq. (6.2).

Let $E_{n}(k), k=1,2, \ldots, 2^{n}$ be the zeros of $\tau_{n}(E)$ in increasing order. Such an ordering is possible, because of

Lemma 13. The zeros of $\tau_{n}$ are real and simple,

$$
E_{n}(1)<E_{n}(2)<\ldots<E_{n}\left(2^{n}\right) .
$$

Moreover, $E_{n}(k)=E_{m}(l)$ if and only if $n=m$ and $k=l$. 
Proof. The first part follows by analogy from the Floquet theory of second order differential equations [24]. In the present case the complete proof is obtained by counting the - real and simple - eigenvalues of the Dirichlet problem: Denote the zeros of $\psi^{0}\left(2^{m}\right)$ by

$$
e_{m}(1)<e_{m}(2)<\ldots<e_{m}\left(2^{m}-1\right)
$$

Then

$$
\left\{e_{m}(k)\right\}_{k=1}^{2^{m-1}}=\bigcup_{n=0}^{m-1}\left\{E_{n}(k)\right\}_{k=1}^{2^{n}}
$$

can be seen from Eq. (3.29). Equating the cardinalities we find the result.

Let us compute the derivative of $\tau_{n+1}(E)$ at a zero of $\tau_{n}(E)$. Equation (3.23) yields

$$
\tau_{n+1}^{\prime}=\left(2 \tau_{n}^{\prime}-\lambda_{n}^{\prime}\right) \tau_{n}-\lambda_{n} \tau_{n}^{\prime}=-\lambda_{n} \tau_{n}^{\prime} \neq 0
$$

Indeed, the preceding lemma and Eq. (3.25) imply that neither $\tau_{n}^{\prime}$ nor $\lambda_{n}$ vanishes if $\tau_{n}=0$. This leads to

Proposition 10. Each zero $E_{n}(k)$ is at the edge of a spectral gap of $H$ and of $H_{n+m}$ for all $m \geqq 1$.

Proof. Fix $E=E_{n}(k)$. Then $\tau_{n}=0, \tau_{n+1}=-2$ (cf. Lemma 2) and $\tau_{n+1}^{\prime} \neq 0$ [Eq. (6.5)]. Suppose e.g. $\tau_{n+1}^{\prime}<0$, then there exists $\varepsilon>0$ such that

$$
\tau_{n+1}(E)<-2 \text { if } E \in\left(E_{n}(k), E_{n}(k)+\varepsilon\right) .
$$

For all these energies $\left\{\tau_{m}\right\}$ is $U_{1}$-type (see Lemma 4), so that

$$
\left(E_{n}(k), E_{n}(k)+\varepsilon\right) \subset\left(\bigcap_{m=1}^{\infty} \varrho\left(H_{n+m}\right)\right)^{\mathrm{int}} \subset U=\varrho(H) .
$$

On the other hand, $E_{n}(k) \in \bigcap_{m=1}^{\infty} \sigma\left(H_{n+m}\right) \cap \sigma(H)$ which shows that $E_{n}(k)$ is a lower gap-edge for all these operators. For $\tau_{n+1}^{\prime}>0$ we find that it is an upper gapedge.

Theorem 3. For all $R>0$ the spectrum of $H$ is a Cantor set.

Proof. Combining Propositions 9 and 10 one obtains that the gaps of $H$ are dense in the spectrum: For any $E \in \sigma(H)$,

$$
\inf _{E^{\prime} \in U}\left|E-E^{\prime}\right|=0 \text {. }
$$

Therefore, the gaps are dense everywhere.

We keep exploiting Proposition 10 to describe the gap structure in detail.

Proposition 11. The zeros of $\psi^{0}\left(2^{n}\right)$ separate the zeros of $\tau_{n}$,

$$
E_{n}(k)<e_{n}(k)<E_{n}(k+1), \quad k=1, \ldots, 2^{n}-1 .
$$

The two sets of zeros together form the set $\left\{e_{n+1}(k) \mid k=1, \ldots, 2^{n+1}-1\right\}$ of the zeros of $\psi^{0}\left(2^{n+1}\right)$. This leads to the following 
Corollary. For $n \geqq 1$,

$$
E_{n}(k)=e_{n+m}\left(2^{m-1}(2 k-1)\right), \quad \text { all } \quad m \geqq 1 .
$$

Proof. By noticing that in Eq. (6.6) we order, in fact, the zeros of $\psi^{0}\left(2^{n+1}\right)$, we find

$$
E_{n}(k)=e_{n+1}(2 k-1)
$$

and

$$
e_{n}(k)=e_{n+1}(2 k) \text {. }
$$

Replacing in the latter equation $n$ by $n+1$ and $k$ by $2 k-1$ we obtain

$$
E_{n}(k)=e_{n+1}(2 k-1)=e_{n+2}(2(2 k-1))=\ldots
$$

as claimed.

Proof of Proposition 11. We use the fact that $P_{k}(E):=\psi^{0}(k+1)(E)$ form a set of orthogonal polynomials relative to some measure (the spectral measure for $H^{r}$ generated by $\left.\delta_{1}\right)$. By Theorem 3.3 .3 of [21], in the open intervals $\left(-\infty, e_{n}(1)\right)$, $\left(e_{n}(1), e_{n}(2)\right), \ldots,\left(e_{n}\left(2^{n}-1\right),+\infty\right)$ there is at least one zero of $\psi^{0}\left(2^{n+1}\right)$. Since the zeros of this latter are $e_{n}(1), \ldots, e_{n}\left(2^{n}-1\right)$ and $E_{n}(1), \ldots, E_{n}\left(2^{n}\right)$, one obtains

$$
\begin{gathered}
E_{n}(1)<e_{n}(1), \quad e_{n}\left(2^{n}-1\right)<E_{n}\left(2^{n}\right), \\
e_{n}(k)<E_{n}(k+1)<e_{n}(k+1), \quad k=1, \ldots, 2^{n}-2,
\end{gathered}
$$

which is equivalent with Eq. (6.6).

Proposition 12. All the zeros $E_{n}(k)$ are lower gap-edges if $\lambda>0$ and upper gap-edges if $\lambda<0$ for $H$ and for $H_{n+m}, m \geqq 1$ (see Fig. 3).

Proof. According to the proof of Proposition 10, the above claim is verified if

$$
\tau_{n}=0 \text { implies } \operatorname{sgn} \tau_{n+1}^{\prime}=-\operatorname{sgn} \lambda \text {. }
$$

Now for $n=0, \tau_{1}^{\prime}=-\lambda \tau_{0}^{\prime}=-\lambda$, and (6.8) holds true. For $n>0, \tau_{n}$ is a polynomial of even degree, hence

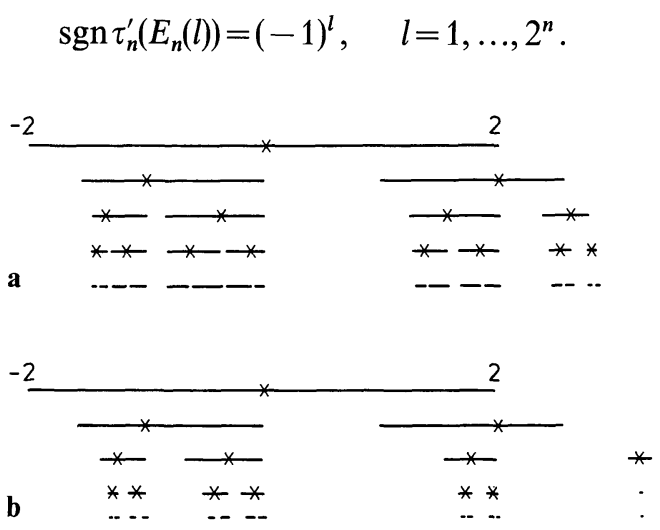

Fig. 3a and b. The spectra of the periodic operators $H_{n}$ for $n=0, \ldots, 4$. $\mathbf{a} \lambda=1, R=0.5$; $\mathbf{b} \lambda=1$, $R=1.5$. The zeros of $\tau_{n}$ are marked by crosses 
On the other hand, $\psi^{0}\left(2^{n}\right)$ is an odd $\left(2^{n}-1\right)$ degree polynomial with principal coefficient 1 for $n>0$. It follows from Proposition 11 that

$$
\operatorname{sgn} \psi^{0}\left(2^{n}\right)\left(E_{n}(l)\right)=(-1)^{l}, \quad l=1, \ldots, 2^{n} .
$$

Replacing $\lambda_{n}$ in Eq. (6.5) by its expression (3.28) gives

$$
\tau_{n+1}^{\prime}=-\lambda R^{n} \psi^{0}\left(2^{n}\right) \tau_{n}^{\prime} \quad \text { if } \tau_{n}=0 .
$$

This together with Eqs. (6.9) and (6.10) proves (6.8).

In this way, we found an everywhere dense set of spectral gaps

$$
I_{n k}= \begin{cases}\left(E_{n}(k), b_{n k}\right), & \lambda>0, \\ \left(a_{n k}, E_{n}(k)\right), & \lambda<0\end{cases}
$$

for $n \geqq 0$ and $k=1, \ldots, 2^{n}$. Equations (6.6) and (6.7) show that the relative position of these gaps is the same as that of the gaps in the classical triadic Cantor set $C$ : The

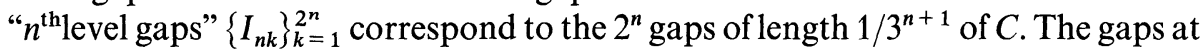
a given level alternate with all those at lower levels.

There is an even deeper similarity with the triadic Cantor set. Let $J_{n k}$ denote the $k^{\text {th }}$ gap of the length $1 / 3^{n+1}$ of $C$ :

$$
J_{01}=\left(\frac{1}{3}, \frac{2}{3}\right), \quad J_{11}=\left(\frac{1}{9}, \frac{2}{9}\right), \quad J_{12}=\left(\frac{7}{9}, \frac{8}{9}\right),
$$

etc. The prototype of singular continuous measures is a measure $d \alpha$ supported by $C$, where $\alpha$ is the Cantor function:

$$
\alpha(x)=\frac{2 k-1}{2^{n+1}} \quad \text { if } \quad x \in J_{n k}
$$

and $\alpha$ extends continuously to $[0,1]$ (cf. [23]). Below we show that a similar equation is valid for the integrated density of states (IDS), where $I_{n k}$ replaces $J_{n k}$ of Eq. (6.12). This does not suggest however that the density of states would be singular continuous!

Let us recall the definition of the IDS. Let $P_{(-\infty, E]}(A)$ denote the spectral projection of the self-adjoint operator $A$ to $(-\infty, E]$. Then the IDS

$$
\mathscr{N}[A](E)=\lim _{L \rightarrow \infty} \frac{1}{L} \sum_{l=1}^{L}\left(\delta_{l}, P_{(-\infty, E]}(A) \delta_{l}\right)
$$

provided that the limit exists. In the present case, the restriction of the summation to $l \geqq 1$ has no effect on the result. For $R<1$, due to the convergence in norm of $H_{n}$ to $H, \mathscr{N}[H]$ exists and

$$
\mathscr{N}[H](E)=\lim _{n \rightarrow \infty} \mathscr{N}\left[H_{n}\right](E) .
$$

On the other hand, one proves easily that for any $R$,

$$
\left|\mathscr{N}\left[H_{n}\right](E)-\mathcal{N}\left[H_{n}^{D}\right](E)\right| \leqq 1 / 2^{n}
$$

uniformly in $E$. [Use the alternance between the zeros of $\tau_{n}-2 \cos \theta$ and those of $\psi^{0}\left(2^{n}\right)$.] Therefore

$$
\mathscr{N}(E)=\lim _{n \rightarrow \infty} \mathscr{N}\left[H_{n}^{D}\right](E)
$$


agrees with the IDS if $R<1$. We show that $\mathcal{N}(E)$ exists also for $R \geqq 1$ and we will consider it as a definition of the IDS.

Theorem 4. For any $R>0$ the IDS exists, it is continuous and

$$
\mathscr{N}(E)=\frac{2 k-1}{2^{n+1}} \text { if } E \in I_{n k} .
$$

Proof. Consider the definition (6.13) for $A=H_{n}^{D}$. Since

$$
p_{n}(l, E)=\left(\delta_{l}, P_{(-\infty, E]}\left(H_{n}^{D}\right) \delta_{l}\right)
$$

is $2^{n}$-periodic in $l$, the limit over $L$ exists and

$$
\begin{aligned}
\mathscr{N}\left[H_{n}^{D}\right](\mathrm{E}) & =\frac{1}{2^{n}} \sum_{l=1}^{2^{n}} p_{n}(l, E) \\
& =\frac{1}{2^{n}} \#\left\{\text { zeros of } \psi^{0}\left(2^{n}\right) \text { in }(-\infty, E]\right\} \\
& =\frac{1}{2^{n}} \max \left\{k \mid e_{n}(k) \leqq E\right\} .
\end{aligned}
$$

By Proposition 11,

$$
\max \left\{k \mid e_{n+1}(k) \leqq E\right\}-2 \max \left\{k \mid e_{n}(k) \leqq E\right\}=0 \text { or } 1 .
$$

As a consequence,

$$
\mathscr{N}\left[H_{n+1}^{D}\right](E) \geqq \mathscr{N}\left[H_{n}^{D}\right](E) .
$$

Now 1 is a uniform upper bound, therefore the limit (6.14) exists for all $E \in \mathbb{R}$. Again, by Proposition 11

thus

$$
\mathscr{N}\left[H_{n+m}^{D}\right]\left(e_{n}(k)\right)=\frac{k}{2^{n}}, \quad \text { all } \quad m \geqq 0,
$$

$$
\mathscr{N}\left(e_{n}(k)\right)=\frac{k}{2^{n}}, \quad n \geqq 1, \quad k=1, \ldots, 2^{n}-1
$$

$\mathscr{N}(E)$ is monotonically increasing, it is between 0 and 1 and its range contains the set (6.16) which is dense in $[0,1]$. Therefore $\mathscr{N}(E)$ is continuous. Finally, from Eqs. (6.16) and (6.7),

$$
\mathscr{N}\left(E_{n}(k)\right)=\mathscr{N}\left(e_{n+1}(2 k-1)\right)=\frac{2 k-1}{2^{n+1}} .
$$

The gap $I_{n k} \subset U$ is disjoint from the set of zeros $\left\{e_{m}(l)\right\}$, so that $\mathscr{N}\left[H_{N}^{D}\right](E)$ is constant in $I_{n k}$ for all $N$. By the convergence, $\mathscr{N}(E)$ is also constant in $I_{n k}$ and, due to the continuity, it equals (6.17), the value at the gap edge.

Now we turn to the problem of the completeness of the gap labelling. A discrete periodic Schrödinger operator with period $L$ may have at most $L-1$ spectral gaps. Therefore the periodic approximant $H_{n}$ may have at most $2^{n}-1$ gaps. 
Proposition 13. $H_{n}$ has $2^{n}-1$ spectral gaps. The set of its gaps is

$$
\left\{I_{n-1, k}^{(1)}\right\}_{k=1}^{2^{n-1}} \cup\left\{I_{n-2, k}^{(2)}\right\}_{k=1}^{2 n-2} \cup \ldots \cup\left\{I_{01}^{(n)}\right\}
$$

where

$$
I_{m k}^{(l)}=\left\{\begin{array}{lll}
\left(E_{m}(k), b_{m k}^{(l)}\right) & \text { if } & \lambda>0, \\
\left(a_{m k}^{(l)}, E_{m}(k)\right) & \text { if } & \lambda<0 .
\end{array}\right.
$$

Proof. Each $E_{m}(k)$ is at the border of a spectral gap $I_{m k}^{(l)}$ for $H_{m+l}$, all $l \geqq 1$ : this was shown in Proposition 10. By simple counting, this statement is equivalent with the claim that $H_{n}$ has no missing gap.

The above proposition establishes a genealogy of a gap $I_{n k}$ of $H: I_{n k}^{(1)}, I_{n k}^{(2)}, \ldots$ are the consecutive precursors of $I_{n k}$. In fact,

$$
I_{n k}=\lim _{l \rightarrow \infty} I_{n k}^{(l)}
$$

in the sense that

$$
\begin{aligned}
& b_{n k}=\lim _{l \rightarrow \infty} b_{n k}^{(l)} \text { if } \lambda>0, \\
& a_{n k}=\lim _{l \rightarrow \infty} a_{n k}^{(l)} \text { if } \lambda<0 .
\end{aligned}
$$

The reader can easily convince himself that the monotonicity property of $U_{1}$-type sequences really implies the convergence (6.18).

Since the family of the gaps of all $H_{n}$ has no other limit points in the above sense, one is tempted to conclude that all the spectral gaps of $H$ have been found and are included in $\left\{I_{n k}\right\}$. This, however, may be wrong. One can imagine, for instance, a gap $(a, b)$ for $H$ of the form

$$
(a, b)=\left(\bigcap_{n} I_{n k_{n}}^{(1)}\right)^{i n t}
$$

The best we can say is

Theorem 5. Let $R<1$ and $(a, b)$ be a spectral gap of $H$. Define $\sum=[-2,2]$ $\cup \bigcup_{m=1}^{\infty} \sigma\left(H_{m}\right)$. If $a \in \sum($ case $\lambda>0)$ or $b \in \sum$ (case $\left.\lambda<0\right)$ then $(a, b)=I_{n k}$ for some $n, k$. Proof. We give it for $\lambda>0$. Clearly $a \in \sigma(H)$. Suppose that $a \in \sigma\left(H_{N}\right)$ for some $N \geqq 0$. Then

$$
a \in \bigcap_{m=0}^{\infty} \sigma\left(H_{N+m}\right),
$$

otherwise we would get $a \in U$ from Lemma 4. Any $\sigma\left(H_{n}\right)$ is composed of $2^{n}$ closed intervals. Let $B_{N+m}$ denote the interval of $\sigma\left(H_{N+m}\right)$ containing $a$. The upper border $u_{N+m}$ of $B_{N+m}$ cannot fall in $(a, b)$ : Indeed, it is in $\sigma(H)$, because $u_{N+m}$ is a lower gapedge for $H_{N+m}$ and thus, by Proposition 13, $u_{N+m}=E_{n}(k)$ for suitable $n, k$. If $B_{N+m} \supset(a, b)$ for all $m \geqq 0$, then $(a, b) \subset \bigcap_{m=1}^{\infty} \sigma\left(H_{N+m}\right) \subset \sigma(H)[$ cf. Eq. (5.23)] which is a contradiction. In this way, there exist $m, n, k: a=u_{N+m}=E_{n}(k)$ implying $(a, b)=I_{n k}$. 
Let us remark the parallelism between Theorem 5 and Proposition 7. In both cases the knowledge that $\bigcup \sigma\left(H_{n}\right)$ covers $\sigma(H)$ would mean a great simplification: $S=\emptyset$ and the completeness of the gap labelling for $R<1$.

\section{Lyapunov Exponent}

For $x \geqq 1$ integer let

$$
T(x)=A_{x} A_{x-1} \ldots A_{1},
$$

where $A_{k}$ are the transfer matrices defined in Eq. (3.10). Take e.g. the matrix norm

$$
\|T\|=\max _{i=1,2}\left\{\left|T_{1 i}\right|+\left|T_{2 i}\right|\right\} \text {. }
$$

Note that $\operatorname{det} T(x)=1$, hence $\|T(x)\| \geqq 1$ and

$$
a(x)=\ln \|T(x)\| \geqq 0 .
$$

The Lyapunov exponent is defined as $\lim _{x \rightarrow \infty} a(x) / x$ provided that the limit exists. Since

$$
\|T(x)\|=\sup _{\left\|\Psi_{0}\right\|=1}\left\|\Psi_{x}\right\|, \quad\left\|\Psi_{x}\right\|=|\psi(x+1)|+|\psi(x)|
$$

[cf. Eq. (3.14)], the Lyapunov exponent yields the maximal rate of increase of wave vectors as $x \rightarrow \infty$. In our case, $x \rightarrow-\infty$ gives the same result. Let

$$
\gamma_{m}=a\left(2^{m}\right) / 2^{m}=\ln \left\|M_{m}\right\| / 2^{m}
$$

Proposition 14. $\gamma=\lim _{m \rightarrow \infty} \gamma_{m}$ exists and

$$
\limsup _{x \rightarrow \infty} a(x) / x=\gamma .
$$

Proof. Taking the norm of Eq. (3.15) and dividing by $2^{m+1}$ we obtain

$$
\gamma_{m+1} \leqq \gamma_{m}+\Delta_{m} / 2^{m+1}
$$

with

$$
\Delta_{m}=\ln \left(1+|\lambda| R^{m}\right)
$$

We define a sequence $x_{m}$ by setting

$$
\gamma_{m}=x_{m}+\sum_{k=0}^{m-1} \Delta_{k} / 2^{k+1}
$$

Replacing this into (7.5) we obtain

$$
x_{m+1} \leqq x_{m}
$$

For any $R>0, \sum_{k=0}^{\infty} \Delta_{k} / 2^{k+1}$ is convergent. By Eq. (7.3) $\gamma_{m} \geqq 0$, therefore

$$
x_{m} \geqq-\sum_{k=0}^{\infty} \Delta_{k} / 2^{k+1}>-\infty
$$


and $\lim x_{m}$ exists. Thus,

$$
\gamma=\lim \gamma_{m}=\lim x_{m}+\sum_{k=0}^{\infty} \Delta_{k} / 2^{k+1} \geqq 0
$$

exists as well. Let now $x \geqq 0$ integer. In diadic representation,

$$
x=\sum_{k \geqq 0} x_{k} 2^{k}, \quad x_{k} \in\{0,1\} .
$$

Due to the symmetry (2.1) of the potential,

$$
T(x)=T\left(\sum_{k=0}^{n} x_{k} 2^{k}\right) T\left(\sum_{k>n} x_{k} 2^{k}\right)
$$

for any $n \geqq 0$. With a repeated application of this factorization we get, by setting $T(0)=\mathbb{1}$,

$$
T(x)=T\left(x_{0} 2^{0}\right) T\left(x_{1} 2^{1}\right) \ldots .
$$

After taking the norm, passing to the logarithm and dividing by $x$ we find

$$
\frac{a(x)}{x} \leqq \frac{1}{x} \sum_{k \geqq 0} a\left(x_{k} 2^{k}\right)=\frac{1}{x} \sum_{k \geqq 0} x_{k} a\left(2^{k}\right) .
$$

Let $\varepsilon>0$ be arbitrary, then there exists an integer $n$ such that $\gamma_{k} \leqq \gamma+\varepsilon$ if $k>n$. We can continue the estimate (7.9) as

$$
\frac{a(x)}{x} \leqq \frac{1}{x} \sum_{k=0}^{n} x_{k} a\left(2^{k}\right)+\frac{1}{x} \sum_{k>n} x_{k} 2^{k} \gamma_{k} \leqq \gamma+\varepsilon+\frac{1}{x} \sum_{k=0}^{n} a\left(2^{k}\right) .
$$

Take now the limits $x \rightarrow \infty$ and then $\varepsilon \rightarrow 0$ to find

$$
\limsup _{x \rightarrow \infty} \frac{a(x)}{x} \leqq \gamma
$$

However, $\gamma=\lim a\left(2^{n}\right) / 2^{n}$, so that $(7.4)$ is verified.

Theorem 6. The Lyapunov exponent exists and is zero: in $\sigma(H)$ if $R \geqq 1$, and in $\sigma(H) \backslash S$ if $R<1$.

Proof. $\sigma(H)$ for $R \geqq 1$ and $\sigma(H) \backslash S$ for $R<1$ agree with $B$, see Theorems 1 and 2 . According to the former proposition, we have to show that $\gamma(E)=0$ if $E \in B$, i.e. $\left\{\tau_{m}(E)\right\}$ is bounded.

We can express $M_{m}^{2}$ from the characteristic equation of $M_{m}$ and insert it into Eq. (3.15):

$$
M_{m+1}=\left(\begin{array}{cc}
1 & -\lambda R^{m} \\
0 & 1
\end{array}\right)\left(\tau_{m} M_{m}-\mathbb{1}\right) .
$$

Let $\tau \geqq\left|\tau_{m}\right|$, all $m$. In Eq. (7.10) let us take the norm then the logarithm and finally divide by $2^{m+1}$. We get

$$
0 \leqq \gamma_{m+1} \leqq \frac{\ln \left[(1+\tau)\left(1+|\lambda| R^{m}\right)\right]}{2^{m+1}}+\frac{1}{2} \gamma_{m} .
$$


Letting $m$ go to infinity, the inequality becomes

which shows that $\gamma=0$.

$$
0 \leqq \gamma \leqq \frac{1}{2} \gamma
$$

Let us recall that for $R \geqq 1$ the spectrum is purely singular continuous, so that the theorem implies singular spectral measures and zero Lyapunov exponent.

\section{Asymptotic Behaviour of the Gap-Edge States}

Apart from the global information given by the Lyapunov exponent, more details can be obtained about the asymptotic behaviour of the solutions of $H \psi=E \psi$ with $E$ being a zero of a $\tau_{m}$. As we have seen in Sect. 6, such an energy is a lower (if $\lambda>0$ ) or upper (if $\lambda<0$ ) gap boundary. From the Floquet theory it is known that among the gap-edge states of any one dimensional periodic Schrödinger equation there is a unique [up to factor] periodic or antiperiodic solution, and the others increase linearly. We may compare this with

Proposition 15. Let $\tau_{m}(E)=0$ and $\psi$ be a solution of $H \psi=E \psi$.

(i) If $\psi(0)=0$ then $\psi\left(k+2^{m+1}\right)=-\psi(k)$ for all $k \in \mathbb{Z}$.

(ii) If $\psi(0) \neq 0$ then $\psi\left(2 l \cdot 2^{m}\right)=(-1)^{l} \psi(0)$ and

$$
\psi\left((2 l+1) 2^{m}\right) \underset{l \rightarrow \infty}{\approx}-\psi(0) \lambda_{m}(-1)^{l} \cdot \begin{cases}\frac{2}{2-R} l, & R<2, \\ l \cdot \log _{2} l, & R=2, \\ \frac{R^{2}}{2(R-1)(R-2)} l^{\log _{2} R}, & R>2 .\end{cases}
$$

Proof. According to the assumption,

and

$$
0=\tau_{m}=\alpha_{m, 0}=\alpha_{m}(2 l+1), \text { all } l \in \mathbb{Z}
$$

$$
\psi\left(2 l \cdot 2^{m}\right)=-\psi\left((2 l-2) 2^{m}\right)=\ldots=(-1)^{l} \psi(0),
$$

see Eqs. (3.5), (3.8), and (3.11). Set at first $\psi(0)=0$, then $\psi\left(l \cdot 2^{m+1}\right)=0$, all $l \in \mathbb{Z}$, and $\psi$ does not depend on the value of the potential at the sites $l \cdot 2^{m+1}$. Thus, $\psi$ solves the $2^{m+1}$-periodic Schrödinger equation

$$
H_{m+1} \psi=E \psi \text {. }
$$

By Lemma 2, $\tau_{m+1}=-2$, so that (8.3) has an, up to factor, unique antiperiodic solution [24]. From Eqs. (2.1) and (2.2)

$$
V_{n}\left(l \cdot 2^{n}-k\right)=V_{n}\left(l^{\prime} \cdot 2^{n}+k\right)
$$

for $l, l^{\prime} \in \mathbb{Z}$ and $k=1, \ldots, 2^{n}-1$. Writing Eq. (8.4) for $n=m+1$ one sees easily that $\psi$ is in fact antiperiodic.

Let now $\psi(0) \neq 0$. Then Eq. (8.2) shows that $\psi$ is bounded oscillating at the sites $2 l \cdot 2^{m}$. On the other hand, from Eqs. (3.11) and (8.2),

$$
\begin{aligned}
\psi\left((2 l+1) 2^{m}\right) & =\alpha_{m}(2 l) \psi\left(2 l \cdot 2^{m}\right)-\psi\left((2 l-1) 2^{m}\right) \\
& =\alpha_{m}(2 l)(-1)^{l} \psi(0)-\psi\left((2 l-1) 2^{m}\right),
\end{aligned}
$$


and after repeated application one obtains

$$
\psi\left((2 l+1) 2^{m}\right)=\psi\left(2^{m}\right)+(-1)^{l} \psi(0) \sum_{k=1}^{l} \alpha_{m}(2 k) .
$$

Replacing $\alpha_{m}(2 k)$ by its expression (3.31) and using $\tau_{m}=0$ this equation becomes

$$
\psi\left((2 l+1) 2^{m}\right)=\psi\left(2^{m}\right)-\psi(0) \lambda_{m}(-1)^{l} \sum_{k=1}^{l} f(\operatorname{ord}(2 k)) .
$$

The density of integers of order $m$ among the even numbers is $1 / 2^{m}$ [cf. Eq. (2.8)], therefore

$$
\begin{aligned}
\sum_{k=1}^{l} f(\operatorname{ord}(2 k)) & =\sum_{m=1}^{\left[\log _{2} 2 l\right]} f(m) \sum_{k=1}^{l} \delta_{\operatorname{ord}(2 k), m} \\
& \underset{l \rightarrow \infty}{\approx} l \sum_{m=1}^{\left[\log _{2} 2 l\right]} f(m) / 2^{m} .
\end{aligned}
$$

Setting $f(m)=\sum_{k=0}^{m-1} R^{k}$, Eq. (8.1) follows after simple calculation.

That something happens at $R=1$, can be noticed only by looking at the corrections to the asymptotic formula (8.1). These can still be obtained from Eq. (8.6) and are respectively of the order of $1 / l, \log _{2} l$ and $l^{\log _{2} R}$ for $R<1, R=1$ and $1<R<2$.

\section{An Explicit Formula for the Green's Function}

We know that the diagonal part of the Green's function

$$
G_{z}(x, y)=\left(\delta_{z},(H-z)^{-1} \delta_{y}\right), \quad x, y \in \mathbb{Z},
$$

is limit periodic when $R<1$, see the remark subsequent to Lemma 9. This implies that it should be possible to write

$$
G_{z}(x, x)=\sum_{n=0}^{\infty} G_{z}^{(n)}(x, x),
$$

where $G_{z}^{(n)}(x, x)$ is a function of period $2^{n}$ of the variable $x$.

We are now going to derive such a formula which will give an "explicit" solution for the Green's function. At this stage, however, we will make no claim of rigor, and our expression will be a formal one.

Let us define the function $F(x, \theta)$ by

$$
G_{z}(x, x+r)=\int_{0}^{2 \pi} \frac{d \theta}{2 \pi} F(x, \theta) e^{i \theta r} .
$$

It should satisfy the linear equation

$$
e^{-i \theta} F(x+1, \theta)+e^{i \theta} F(x-1, \theta)+(V(x)-z) F(x, \theta)=1 .
$$

Note that if the potential is almost periodic then so is $F$, with the same frequency module. Our potential has the form

$$
V(x)=\sum_{n=0}^{\infty} v_{n}(x),
$$


where $v_{n}(x)$ is of period $2^{n}$,

$$
\begin{aligned}
& v_{0}(x)=\frac{\lambda}{1-R}, \\
& v_{n}(x)=-\frac{\lambda}{1-R} R^{n-1} \sum_{j} \delta_{x, 2^{n-1}(2 j+1)}, n \geqq 1 .
\end{aligned}
$$

We look for a solution of (9.3) of the form

$$
F(x, \theta)=\sum_{n=0}^{\infty} f_{n}(x, \theta)
$$

with $f_{n}(x)$ of period $2^{n}$. Using the explicit form (9.5) of the potential one can see that

$$
V(x) F(x)=V(x) F(0)+\sum_{n=0}^{\infty}\left[f_{n}(x)-f_{n}(0)\right] \sum_{j=0}^{n} v_{j}(x) .
$$

Equation (9.3) then gives after some computation

$$
f_{0}(x)=\frac{v_{0}(0) F(0)-1}{z-2 \cos \theta}
$$

and for $\phi_{n}(x)=f_{n}(x)-f_{n}(0)$,

$$
\phi_{n}(x)=\sum_{y=0}^{2^{n}-1}\left[H_{n}(\theta)-z\right]^{-1}(x, y)\left\{(z-2 \cos \theta) f_{n}(0)-v_{n}(y) F(0)\right\} .
$$

Here $H_{n}(\theta)$ is the Hamiltonian defined by

$$
\left(H_{n}(\theta) \phi\right)(x)=e^{-i \theta} \phi(x+1)+e^{i \theta} \phi(x-1)+W_{n}(x) \phi(x)
$$

in $\left[0,2^{n}-1\right]$ with periodic boundary condition, and

$$
W_{n}(x)=\sum_{j=0}^{n} v_{j}(x)
$$

Defining

$$
K_{n}(x)=\sum_{y=0}^{2^{n}-1}\left(H_{n}(\theta)-z\right)^{-1}(x, y)
$$

and

$$
L_{n}(x)=\sum_{y=0}^{2^{n}-1}\left(H_{n}(\theta)-z\right)^{-1}(x, y) v_{n}(y),
$$

we see that for $n \geqq 1$,

$$
f_{n}(x)=F(0)\left\{\frac{L_{n}(0)}{K_{n}(0)}\left[\frac{1}{z-2 \cos \theta}+K_{n}(x)\right]-L_{n}(x)\right\} .
$$

Inserting this expression and (9.8) into the definition (9.6) we get the following expression for $F(0)$,

$$
F(0)^{-1}=2 \cos \theta+\sum_{n=1}^{\infty} \frac{L_{n}(0)}{K_{n}(0)}+v_{0}-z .
$$


This "solves," in principle, the problem we posed. We now express $F(0)$ in a more explicit form. For this, let us note that $\phi_{n}(x)$ is the solution of the inhomogeneous equation,

$$
\begin{aligned}
& e^{-i \theta} \phi_{n}(x+1)+e^{i \theta} \phi_{n}(x-1)+\left[W_{n}(x)-z\right] \phi_{n}(x) \\
& \quad=(z-2 \cos \theta) f_{n}(0)-v_{n}(x) F(0)
\end{aligned}
$$

with $\phi_{n}(0)=0$. Since

$$
\begin{gathered}
W_{n}(2 x+1)=v_{n}(2 x+1)=0, \\
W_{n}(2 x)=\lambda+R W_{n-1}(x), \\
v_{n}(2 x)=R v_{n-1}(x),
\end{gathered}
$$

when $n \geqq 2$, we can make a renormalization transformation of this equation by eliminating odd sites. For $n \geqq 2$, we get

$$
\begin{aligned}
& e^{-i 2 \theta} \phi_{n}(2 x+2)+e^{2 i \theta} \phi_{n}(2 x-2)+\left[W_{n-1}^{\prime}(x)-z^{\prime}\right] \phi_{n}(2 x) \\
& \quad=\left(z^{2}-4 \cos ^{2} \theta\right) f_{n}(0)-v_{n-1}^{\prime}(x) F(0)
\end{aligned}
$$

Here $z^{\prime}=z^{2}-2-\lambda z$ and $W_{n-1}^{\prime}$ and $v_{n-1}^{\prime}$ are the same as $W_{n-1}$ and $v_{n-1}$ except that $\lambda$ has been replaced by $\lambda^{\prime}=z R \lambda$. The variables $z$ and $\lambda$ undergo the same transformation as $\tau_{m}$ and $\lambda_{m}$ earlier, see Eq. (3.23).

The condition $\phi_{n}(0)=0$ gives now

$$
\frac{f_{n}(0, \theta ; \lambda, z)}{F(0, \theta ; \lambda, z)}=\frac{1}{z^{2}-4 \cos ^{2} \theta} \frac{L_{n-1}\left(0,2 \theta ; \lambda^{\prime}, z^{\prime}\right)}{K_{n-1}\left(0,2 \theta ; \lambda^{\prime}, z^{\prime}\right)}
$$

Let us define

$$
g_{n}(\theta ; \lambda, z)=\frac{f_{n}(0, \theta ; \lambda, z)}{F(0, \theta ; \lambda, z)}(z-2 \cos \theta) .
$$

Then from Eq. (9.14),

$$
g_{n}(\theta ; \lambda, z)=\frac{L_{n}(0, \theta ; \lambda, z)}{K_{n}(0, \theta ; \lambda, z)},
$$

and Eq. (9.18) transforms into the recursion formula

$$
g_{n}(\theta ; \lambda, z)=\frac{1}{z+2 \cos \theta} g_{n-1}\left(2 \theta ; \lambda^{\prime}, z^{\prime}\right)
$$

Using

$$
g_{0}(\theta ; \lambda, z)=\frac{\lambda}{1-R},
$$

after $n$ steps of iteration we obtain

$$
g_{n}(\theta ; \lambda, z)=\frac{\lambda^{(n)}}{1-R} \prod_{m=0}^{n-1} \frac{1}{z^{(m)}+2 \cos 2^{m} \theta} .
$$


Since $\left\{z^{(m)}, \lambda^{(m)}\right\}$ satisfy the recurrence equations (3.23), we may keep the earlier notations,

$$
z=\tau_{0}, \quad z^{(m)}=\tau_{m}, \quad \lambda^{(m)}=\lambda_{m} .
$$

With this identification we arrive at

$$
F(0)^{-1}=2 \cos \theta-z+\frac{\lambda}{1-R}+\frac{1}{1-R} \sum_{n=1}^{\infty} \lambda_{n} \prod_{m=0}^{n-1} \frac{1}{\tau_{m}+2 \cos 2^{m} \theta} .
$$

For the Green's function this gives

$G_{z}(0, r)=\int_{0}^{2 \pi} \frac{d \theta}{2 \pi}\left[2 \cos \theta-z+\frac{\lambda}{1-R}+\frac{1}{1-R} \sum_{n=1}^{\infty} \lambda_{n} \prod_{m=0}^{n-1}\left(\tau_{m}+2 \cos 2^{m} \theta\right)^{-1}\right]^{-1} \times e^{i \theta r}$,

a formula in terms of the renormalized energies, $\tau_{n}$ and coupling constants, $\lambda_{n}$. To give a rigorous meaning to this expression one should analyze the $R G$ transformation on the complex plane, a task which seems not to be easy.

\section{References}

1. Fröhlich, J., Spencer, T.: Absence of diffusion in the Anderson tight binding model for large disorder or low energy. Commun. Math. Phys. 88, 151-184 (1983)

2. Huberman, B.A., Kerszberg, M.: Ultradiffusion: the relaxation of hierarchical systems. J. Phys. A 18, L331-L 336 (1985)

3. Teitel, S., Domany, E.: Dynamical phase transitions in hierarchical structures. Phys. Rev. Lett. 55, 2176-2179 (1986)

4. Maritan, A., Stella, A.L.: Exact renormalization group approach to ultradiffusion in a hierarchical structure. J. Phys. A 19, L269-L273 (1986) and Exact renormalization group for dynamical phase transition in hierarchical structures. Phys. Rev. Lett. 56, 1754 (1986)

5. Teitel, S.: Transition to anomalous relaxation; localization in a hierarchical potential. University of Rochester preprint (1988)

6. Jona-Lasinio, G., Martinelli, F., Scoppola, E.: Quantum particle in a hierarchical potential with tunneling over arbitrarily large scales. J. Phys. A 17, L635-L638 (1984); Multiple tunnelings in $d$-dimensions: a quantum particle in a hierarchical potential. Ann. Inst. Henri Poincaré 42, 73-108 (1985)

7. Roman, H.E.: Electronic properties of a one-dimensional hierarchical system. Phys. Rev. B 36, 7173-7176 (1987)

8. Ceccato, H.A., Keirstead, W.P., Huberman, B.A.: Quantum states of hierarchical systems. Phys. Rev. A 36, 5509-5512 (1987)

9. Ceccato, H.A., Keirstead, W.P.: Critical eigenfunctions in a quantum hierarchical system. J. Phys. A 21, L75-L 83 (1988)

10. Schneider, T., Wurtz, D., Politi, A., Zannetti, M.: Schrödinger problem for hierarchical heterostructures. Phys. Rev. B 36, 1789-1792 (1987)

11. Livi, R., Maritan, A., Ruffo, S.: The spectrum of a 1-D hierarchical model. J. Stat. Phys. 52, 595-608 (1988)

12. Cycon, H.L., Froese, R.G., Kirsch, W., Simon, B.: Schrödinger operators. Berlin, Heidelberg, New York: Springer 1987, Chaps. 9 and 10

13. Bellissard, J.: Almost periodicity in solid state physics and $C^{*}$ algebras. Talk given at the Harald Bohr Centenary Conference on Almost Periodic Functions, April 25, 1987

14. Pearson, D.B.: Singular continuous measures in scatterings theory. Commun. Math. Phys. 60, 13-36 (1978) 
15. Avron, J., Simon, B.: Singular continuous spectrum for a class of almost periodic Jacobi matrices. Bull. AMS 6, 81-86 (1982)

16. Casdagli, M.: Symbolic dynamics for the renormalization map of a quasiperiodic Schrödinger equation. Commun. Math. Phys. 107, 295-318 (1986)

17. Sütö, A.: The spectrum of a quasiperiodic Schrödinger operator. Commun. Math. Phys. 111, 409-415 (1987)

18. Kohmoto, M., Kadanoff, L.P., Tang, C.: Localization problem in one dimension: mapping and escape. Phys. Rev. Lett. 50, 1870-1872 (1983)

19. Ostlund, S., Pandit, R., Rand, D., Schellnhuber, H.J., Siggia, E.D.: One-dimensional Schrödinger equation with an almost periodic potential. Phys. Rev. Lett. 50, 1873-1876(1983)

20. Mahler, K.: p-adic numbers and their functions. Cambridge: Cambridge University Press 1981

21. Szego, G.: Orthogonal polynomials. Providence, RI: American Mathematical Society 1939

22. Pastur, L.: Spectral properties of disordered systems in the one-body approximation. Commun. Math. Phys. 75, 179-196 (1980); see also ref. 12

23. Reed, M., Simon, B.: Methods of modern mathematical physics, Vol. I. New York: Academic Press 1980

24. Eastham, M.S.P.: The spectral theory of periodic differential equations. Scottish Academic Press 1973

25. Bellissard, J., Scoppola, E.: The density of states for almost periodic Schrödinger operators and the frequency module: a counterexample. Commun. Math. Phys. 85, 301-308 (1982)

26. Private communication by B. Simon

Communicated by J. Fröhlich

Received August 30, 1988 
